\title{
Model Augmented Reality Curriculum
}

\author{
Mikhail Fominykh* \\ Molde University College \\ Molde, Norway \\ mikhail.fominykh@himolde.no \\ Mark Billinghurst \\ University of South Australia \\ Adelaide, Australia \\ mark.billinghurst@unisa.edu.au \\ Eleni Mangina \\ University College Dublin \\ Dublin, Ireland \\ eleni.mangina@ucd.ie
}

\author{
Fridolin Wild ${ }^{*}$ \\ The Open University \\ Milton Keynes, United Kingdom \\ f.wild@open.ac.uk \\ Lisandra S. Costiner \\ Merton College, University of Oxford \\ Oxford, United Kingdom \\ lia.costiner@merton.ox.ac.uk \\ Judith Molka-Danielsen \\ Molde University College \\ Molde, Norway \\ judith.molka-danielsen@himolde.no
}

\author{
Ralf Klamma* \\ RWTH Aachen University \\ Aachen, Germany \\ klamma@dbis.rwth-aachen.de \\ Andrey Karsakov \\ ITMO University \\ Saint Petersburg, Russia \\ karsakov@itmo.ru \\ Ian Pollock \\ California State University East Bay \\ Hayward, United States \\ ian.pollock@csueastbay.edu
}

\author{
Marius Preda \\ Institute MINES-Telecom \\ Paris, France \\ marius.preda@it-sudparis.eu
}

\author{
Aljosa Smolic \\ Trinity College Dublin \\ Dublin, Ireland \\ smolica@tcd.ie
}

\begin{abstract}
Augmented Reality (AR) is a rapidly growing field in information and communication technologies, drawing increasing numbers of professionals. Higher education institutions, however, are struggling to keep abreast of its development and to train specialists quickly, providing few courses which sufficiently align with the needs of industry. In addition to this, the field is developing so rapidly that existing courses struggle to keep pace. They also often focus too narrowly on specifics to allow for the building of the formative foundations of AR education. This paper aims to address this need by proposing a blueprint curriculum in Computer Science Education for teaching AR in universities at two levels, foundations and advanced. To begin, we survey the state of the art, identifying common needs and problems in existing courses which focus on AR. We then detail a skills framework comprised of 12 groups of skills suitable to meet industry needs, and built upon it two model lesson plans for a foundation and an advanced course. We conclude with a discussion of assessment techniques and curricular design options of embedding such coursework into existing academic programs and a forecast of the future of this academic field.
\end{abstract}

${ }^{*}$ Working group leaders

Permission to make digital or hard copies of all or part of this work for personal or classroom use is granted without fee provided that copies are not made or distributed for profit or commercial advantage and that copies bear this notice and the full citation on the first page. Copyrights for components of this work owned by others than ACM must be honored. Abstracting with credit is permitted. To copy otherwise, or republish, to post on servers or to redistribute to lists, requires prior specific permission and/or a fee. Request permissions from permissions@acm.org.

ITiCSE-WGR '20, June 17-18, 2020, Trondheim, Norway

(c) 2020 Association for Computing Machinery.

ACM ISBN 978-1-4503-8293-9/20/06 . .\$15.00

https://doi.org/10.1145/3437800.3439205

\section{CCS CONCEPTS}

- Social and professional topics $\rightarrow$ Model curricula; Computer science education; $\bullet$ Human-centered computing $\rightarrow$ Mixed / augmented reality.

\section{KEYWORDS}

Software Engineering, Augmented Reality, Curriculum

ACM Reference Format:

Mikhail Fominykh, Fridolin Wild, Ralf Klamma, Mark Billinghurst, Lisandra S. Costiner, Andrey Karsakov, Eleni Mangina, Judith Molka-Danielsen, Ian Pollock, Marius Preda, and Aljosa Smolic. 2020. Model Augmented Reality Curriculum. In 2020 ITiCSE Working Group Reports (ITiCSE-WGR '20), June 17-18, 2020, Trondheim, Norway. ACM, New York, NY, USA, 19 pages. https://doi.org/10.1145/3437800.3439205

\section{INTRODUCTION}

A major challenge faced by emerging fields is that they are always taught by people who were educated in other disciplines. One needs only to look at Computer Science (CS). In the early 1980s, there were limited numbers of CS courses offered by universities across the world. Teachers who taught this material often came from the disciplines of engineering, mathematics, and physics. These teachers had not been taught CS and, on occasion, had little prior experience in designing and programming computers. We face a similar situation today in the emerging field of Augmented Reality (AR).

As we step forward again, educators cannot choose piecemeal from the offshoots and specializations of a general CS curricula which does not suit the learning objectives of the new emergent field of AR. It is, therefore, crucial to identify and draw together the skills required to address the new learning objectives for AR.

In the design of immersive experiences and their associated products across multi-modal platforms, it is important to combine 
both creative and technical skills. Few of the available teachers may have the specialised and diverse backgrounds needed to cover such a range of expertise within the new field. Given such limited skills, AR course designs are typically created as inadequate derivations of courses delivered previously. This underscores the need for a model AR curriculum.

AR refers to enhancing human perception with additional, artificially generated sensory input to create a new experience including, but not restricted to, enhancing human vision by combining natural and digital content [33]. AR is a significant part of the next wave of Personal Computing [29], ubiquitous, pervasive, wearable, omnipresent, and equipped with the ability to treat reality like a medium. Creating high quality user experience requires specialists that are educated in the design, implementation, deployment, and evaluation of applications that would incorporate rich artificial content in real-world scenarios [6].

$\mathrm{AR}$ is a rapidly growing field amongst information and communication technologies [32]. The evolution of AR technology is accelerating, requiring an increasing number of skilled professionals. Digital transformation triggered by emerging technologies will likely impact the workforce as a whole, so AR development skills are critical to address these new demands.

At the same time, it is a significant challenge to find a suitable university course or school that is teaching AR development skills at the level required to meet industry needs. Students interested in a career in this field are often advised instead to choose a technical degree such as engineering or design, to learn AR-specific skills independently or in the workplace. However, the ideal background for AR involves skills drawn from a range of different disciplines such as engineering, psychology, design and management, among others.

At the time of writing this proposal for a model curriculum, educational offers in AR are very fragmented and scarce, usually combined with Human-Computer Interaction (HCI) or Virtual Reality (VR), following the ACM and IEEE Computer Society curricular recommendations ${ }^{1}$. Five disciplines are covered by these recommendations, all impacting AR education: Computer Engineering, Computer Science, Information Systems, Information Technology, and Software Engineering. Additionally, the recommendations are for the Bachelors and the Masters level as well as for K12 and continuing education. The primary audience is the United States, but some recommendations are available in Chinese as well, and recommendations for MBAs are targeting a global market.

The 2016 Computer Engineering [1] and the 2017 Information Technology [2] curricular recommendations comment on AR as follows, "the computer engineering/IT curriculum should allow exploration of new inventions that have yet to emerge as viable technologies. For example, teachers might encourage exploration of ways in which a computer engineer would design environments involving augmented reality and virtual worlds or ways in which big data and data analytics might affect the work of a computer engineer." The 2013 computer science Bachelor's education report [12] includes AR as part of a human-computer interaction course. The other subject recommendation reports do not mention AR at all.

\footnotetext{
${ }^{1}$ https://www.acm.org/education/curricula-recommendations
}

With this work, we seek to remedy this situation. Our aim is therefore twofold. First, we survey the state of the art in teaching AR and summarize features and highlight issues of current educational offers. Secondly, we describe a model curriculum that incorporates best practices and addresses existing challenges.

In assessing the state of the art in teaching AR, we reviewed existing course offers which focus solely on this topic as well as courses that include this topic as a key component. We collected information about existing teaching material employed both inside and outside of academia. We identified printed and online textbooks, web-based and video step-by-step tutorials, video lectures and slide decks, as well as campus and online courses. We also searched academic publications on teaching AR, a search which yielded few results, but which served to further underscore the need for a model curriculum. Based on this survey, we identified the most common issues and gaps in teaching AR.

In developing a model Augmented Reality curriculum, which would enable both educational organizations and individuals to teach the topic, we propose a skills framework. This comprises two lesson plans for a foundation and advanced course and maps the skills to intended learning outcomes. The framework we propose classifies the most valuable skills and those most required by industry into groups. As for the syllabus, we discuss which units the students must learn, should learn, and which are supplementary. Moreover, we discuss assessment regimes and organisational design options of how the courses can be embedded in existing programs.

In this paper, we collated the current educational offers in the field of $\mathrm{AR}$ and analyzed the job market to assess which skills are most valued by employers in filling future jobs [16]. We map the state of AR curricula on offer and identify issues and gaps. We then develop a skills framework and blueprint model for the design of AR curricula that highlights the features, objectives, and approaches that should be considered in addressing current gaps in AR education.

\section{THE SKILLS GAP TO INDUSTRY}

In the introduction, we claim that an increasing number of skilled professionals, trained in AR, is needed. This claim derives from three key surveys of the AR industry which outline desired skills.

A 2019 skill survey was conducted by Burning Glass and Epic games in cooperation with Unreal, which focused on creative and 3D graphic skills, included AR [24]. Their findings affirm that all 3D skills, including those in AR, are under-supplied. The report also emphasises that the number of hybrid jobs that require both creative and technical skills is growing. Their results highlight the desirability of 3D skills, of which AR skills have the highest projected growth in demand (207\% in 10 years). The growth in demand is also reported by Hired that registered a growth of $1400 \%$ in demand for AR/VR engineers during 2019, based on the data from employers and job seekers [19].

Story Futures Academy, UK's National Centre for Immersive Storytelling, also produced a report, which notes a significant gap in immersive or XR (Mixed Reality or both AR and VR) skills based on a survey of the creative industry in the UK [8]. Nearly all surveyed companies (97\%) report missing XR skills, while $65 \%$ of them consider this as a major barrier to their growth. The report also 
identifies a need for both technical and creative skills. The finding stress that the field of XR is interdisciplinary, meaning that teams must work across a range of technologies and creative processes. The private sector is concerned that there is a lack of graduates with relevant skills to meet the demand in the emerging discipline, which can be interpreted as a broken skills pipeline. Formal training is not part of the culture in the creative sector thus propagating the absence of curricular models in AR.

A third report created by the AR-FOR-EU project[16] presents findings from an industry survey, thus focusing on the technical aspects of AR. The study design uses AR job offers to extract relevant skills, incorporating a survey component (of industry representatives) as a follow-up to validate and group these skills. The job market study identified skills required for AR specialists by screening over 100 job announcements on several online job portals in multiple countries. The skills requirements mentioned in these job announcements were grouped into ten categories, with several individual sub-skills in each. Almost all identified skills were rated as highly important, confirming the selection and grouping of skills as relevant to industry.

Since industry needs are often practically oriented, and not necessarily focused on long-term employability of graduates, the applied needs of industry remain in constant tension with the learning objectives of Higher Education. The widespread use of an accepted skills framework and associated model curriculum, however, can provide a basis for a potential convergence.

This review of skills desired by industry forms the basis for the current proposal for a comprehensive framework of 'hard' skills, elaborated in Section 3. The proposal draws upon the findings of the AR-FOR-EU project [16], prompted by the gap in literature on the subject, and extends and refines these findings with input from the working group. One significant difference is that the HCI skills were split into interaction technology skills and evaluation skills, and several of the sub-skills were added, updated, or refined, as described in Section 4.1.

\section{THE STATE OF THE ART IN TEACHING AUGMENTED REALITY}

The topics of teaching AR as part of CS study programs is generally unexplored by the CS Education communities. Research on the topic is very limited. The search for "Augmented reality" to appear anywhere in the proceedings of ACM ITiCSE from 1998 to 2020 returns 16 results $^{2}$. Among these results, 15 papers focus on using AR in educational settings. Only one of them focuses on teaching $\mathrm{AR}$, a working group report by the authors of this work [17].

The same search for "Augmented reality" to appear anywhere in the proceedings of ACM SIG CSE from 1986 to 2020 returns 56 results ${ }^{3}$. These results included those 16 from the previous search. Among the 40 new papers, only one has some relevance to teaching AR [7]. This paper describes a game design course, in which student assignments were to design AR apps.

\footnotetext{
${ }^{2}$ https://dl.acm.org/action/doSearch?AllField=\%22Augmented + Reality $\% 22 \&$ expand $=$ all\&ConceptID $=119483$

${ }^{3}$ https://dl.acm.org/action/doSearch?AllField=\%22Augmented+Reality\%22\&expand= all\&ConceptID $=118177$
}

The available papers on the topic found elsewhere study specific aspects of teaching of AR. For example, [25] explores the feasibility of including the development of VR/AR applications to undergraduate programs and concludes that the students are indeed capable of completing such tasks. Practical challenges for teaching XR are described in [27], highlighting the importance of hands-on experience with special purpose hardware, discussed in the context of the experience of teaching a course on XR.

Higher Education teaching practices in the field of AR and the industry requirements for AR specialists were surveyed in [16] and [20]. The reports highlight the absence of AR courses in study programs of top-ranked universities across the world, identifying only 19 courses taught in 15 universities with the help of searches in study portals. The courses were found to be part of different study programs ranging from classical CS to Media, and from specialized AR programs to Social Sciences. The analysis of their curricula revealed some patterns in the content of the courses and in assessment, but no agreement across universities. Prerequisites for taking these courses ranged from no requirements to knowledge in math, linear algebra, programming, computer vision, computer graphics, interaction design, and AR/VR development (sic!).

Fominykh et al. conclude that the field of AR has a large volume of materials that can be organised in different ways [16]. Moreover, learning outcomes, course objectives, and assessment methods are not consistent, underlining the need for a holistic overview on AR curricula.

Subsequently, we identify and present additional features and the challenges of the existing AR curricula. We are not aiming to be exhaustive and cover all AR courses available, but try to identify specific aspects of good practice and highlight specific common issues people will face when implementing an AR course module into their regular teaching cycle. We therefore used a soft inclusion criteria of AR-specific content covering at least $30 \%$ of a course and the availability of the course description online. We started by conducting a search on a global online aggregator of study programs, Masterportal ${ }^{4}$. The search for a key phrase "Augmented Reality" returned 98 results. For each result, we tried to find an official university web page with course description. After the review of the results, only 10 courses were selected. We then searched online using several major search engines and several key phrases, including combinations of "Augmented Reality", "Mixed Reality", and "Extended Reality" with "Course" and "University". This search resulted in six additional courses that were included. Finally, we supplemented these results with the 19 courses identified in the previous work [16]. After an in-depth review, only 14 of them were included.

Our final list consisted of 31 courses offered by 22 universities. We group these along their disciplinary center of gravity, highlighting the state of affairs in offers in Computer Science, in Design/Media/Communication Studies, in offers situated in specific Application Domains (like medicine), and regarding the few existing specialisations. Additionally, we review online offers and commercial offerings.

\footnotetext{
${ }^{4}$ https://www.mastersportal.com/
} 


\subsection{AR Curricula in Computer Science Programs}

We identified at least three clusters of AR courses taught as part of CS programs at different universities. First, there are AR courses in CS programs that focus on low-level graphics, computer vision and data science skills.

The University of Genoa offers a course Computer Graphics and Augmented Reality ${ }^{5}$. The course combines Computer Graphics (including rendering, GPU, affine and projective geometry, geometric transformations, data-driven programming, and WebGL), Computer Vision (image processing, calculation of disparity and optical flow, camera calibration, Simultaneous Localization and Mapping - SLAM), and AR/VR development (visual perception, AR devices, interaction with virtual objects, visual coherence, occlusion management, and case studies). The same study program also contains courses on Computer Vision, Image Processing, and Machine Learning.

The Augmented Reality course at the Trinity College Dublin ${ }^{6}$ is another example of an AR course in a CS program that focuses on computer vision and graphics. The course covers fundamentals, such as pose estimation and marker-based AR, AR SDKs, and state-of-the-art in AR. The course also covers 3D computer vision, mathematical camera models, camera calibration, and computer vision libraries. Other topics include feature extraction and tracking, warping and blending, panoramic and 360 video. The course also includes the topics of 3D reconstruction, integrating virtual elements into real world views. Small exercises and tutorials are given on software libraries and SDKs. Assessment is based $100 \%$ on a single self-defined final project from the broad area of the course.

Second, there are AR courses in CS programs that focus on application design and high-level AR development skills, also complemented by VR development skills.

The Virtual and Augmented Reality course offered by the Computer and Information Science department at Cornell University combines AR with VR in all their topics, emphasising design and development of XR applications ${ }^{7}$. The course covers the concepts of XR, interaction techniques, human perception, and AR/VR development. The course also introduces the relevant concepts of computer vision, computer graphics, and $\mathrm{HCI}$. The course project is on development of XR applications.

The University of South Australia runs a thirteen week course in Virtual and Augmented Reality for final year undergraduate students in the CS department ${ }^{8}$. The course involves a single two hour lecture a week, with a one hour tutorial. The lectures mostly focus on a high level technology overview, while the tutorials provide hands-on AR/VR development with the Unity game engine. The lectures include content on AR/VR definition and history, the perceptual basis of AR/VR, AR/VR technology overview, 3D user interface design, interaction design for AR/VR, VR systems, AR/VR

\footnotetext{
${ }^{5}$ https://web.archive.org/web/20200913173553/https://unige.it/off.f/2018/ins/27865 html

${ }^{6}$ https://web.archive.org/web/20200913174020/https://www.scss.tcd.ie/modules/ 2019-2020/CS7GV4.AUGMENTED_REALITY.2019-2020.5.SEM202.pdf

${ }^{7}$ https://web.archive.org/web/20200913175117/https://www.cs.cornell.edu/courses/ cs5650/

${ }^{8}$ https://web.archive.org/web/20200913175411/https://my.unisa.edu.au/public/ courseoutline/ViewOutline.aspx?id=28515
}

applications, mobile AR, and research directions in AR/VR. The assessment involves developing three working VR/AR applications, plus reading and presenting an AR/VR research paper.

Third, there are AR courses in CS programs that focus on $H C I$ and interaction design skills. In such programs, AR is usually combined with related fields under the umbrella of XR and advanced interactive technologies.

Human-Computer Interaction: Issues in Mixed and Augmented Reality at Stanford University is a prominent example of a course with a strong $\mathrm{HCI}$ perspective on $\mathrm{AR}^{9}$. The course covers an introduction to XR and $\mathrm{HCI}$ issues in XR, displays, tracking and registration, AR development and SDKs, and authoring tools, rendering, perception, 3D interaction, wayfinding, visualisation and spatial data, application cases, ethical issues, and the future of XR.

The 3D User Interfaces and Augmented Reality course at Columbia University provides an HCI perspective to AR and focuses on designing $3 \mathrm{D}$ user interfaces ${ }^{10}$. The course covers metaphors for 3D user interfaces, 3D perception, displays, tracking, manipulating 3D objects, wayfinding in XR, multimodal interaction, and more. The assessment is based on assignments to evaluate 3D user interfaces, design and development of AR apps with 3D interaction, a design analysis task, and a final XR development group project.

AR courses taught in CS programs provide the technical perspective, focusing on either graphics and vision or AR/VR development or HCI and interaction. They rarely cover, however, all aspects thoroughly. CS programs often focus on almost orthogonal sets of skills which make it impossible for employers to rely on the quality of CS education. Media and design topics are largely absent.

\subsection{AR Curricula in Design, Media and Communication Science Programs}

The Mobile Interaction Design course offered by the Department of Communications and New Media at the National University of Singapore presents a design perspective on $\mathrm{AR}^{11}$. The course is structured around the concepts as situatedness, context, and mobile media in the context of designing for mobile platforms. The course also covers evaluation methodologies and design practices for spatial interaction and AR. The practical part of the course includes the application of design principles and usability goals, developing mobile AR applications.

Another example in this category is the Virtual Reality/Augmented Reality course offered at the International Technological University as part of the Master's program in digital arts ${ }^{12}$. This course focuses on the design and evaluation of XR systems. The topics covered in the course include delivery systems, interaction in XR, psychological aspects, XR evaluation techniques, marker-based AR and tracking, and mobile application development. The courses of the study program include game development, 3D modeling and 3D printing, CG software fundamentals, and Unity platform.

\footnotetext{
${ }^{9}$ https://web.archive.org/web/20200913175920/https://web.stanford.edu/class/ cs $377 \mathrm{~m} /$ schedule.html

${ }^{10}$ https://web.archive.org/web/20200913180523/https://graphics.cs.columbia.edu/ courses/csw4172/

${ }^{11}$ https://web.archive.org/web/20200913181611/https://ivle.nus.edu.sg/V1/ $\mathrm{lms} /$ public/view_moduleoutline.aspx?CourseID=73cc931e-e316-49b7-8dc0$12 \mathrm{f} 05 \mathrm{~b} 31042 \mathrm{~d}$

${ }^{12}$ https://web.archive.org/web/20200913182414/https://www.itu.edu/academics/ digital-arts/curriculum
} 
A final example is the Augmented Reality Design: Principles and Practice course offered in the Film and Media study program by the Department of Media Design and Technology at the University of Bradford ${ }^{13}$. The course focuses on the design and development of marker-based and location-based AR applications, covering the topics of programming, AR development tools, object recognition and tracking, geolocation, multiplatform development, standards and workflows, AR storytelling, creating 3D assets, and ethical issues.

The major shortcoming of the AR courses in the design, media and communication programmes is the lack of CS topics in their curricula. Although the HCI and high-level AR/VR development aspects are often covered in these programmes, many important topics are clearly missing. The challenge is also that these programmes do not include courses that cover basic programming skills, which are usually prerequisite for the AR courses in the CS programs. The target group of the design, media and communication programs requires a different approach to the introduction of technical aspects of AR compared to the students at the CS programmes.

\subsection{Curricula in Application Domains}

There are curricula that deeply integrate AR with its application domain or domains in a single or a series of multidisciplinary courses. One of such application domains is medicine. The Technical University of Munich is known for their course on Medical $\mathrm{AR}^{14}$. The course largely covers AR topics, while the medical application comes as an addition. The AR topics covered by the course are similar to those in the typical CS programs with the focus on computer vision and graphics. The topics include vision and perception, geometry, hardware and sensing, cameras, audio, user interaction, and graphics. The medical aspects covered in the course include the medical application of AR and introductory medical topics.

The Augmented Reality course ${ }^{15}$ at Johns Hopkins University is offered by the laboratory for Computer Aided Medical Procedures that collaborates with the Technical University of Munich. The course also focuses on the medical application of AR. It also covers the AR topics similar to those in the typical CS programs with the focus on computer vision and graphics. The medical components of the course include particular requirements for AR systems in the application domain, tracking and visualization in medical AR, state of the art in medical AR, and specific industrial application of medical AR systems.

The Medical Visualisation course ${ }^{16}$ offered as a part of a joint program at the University of Glasgow and Glasgow School of Art is another example of integrating AR and its medical application. The course is a part of a more balanced study program that focuses equally on visualisation technologies, including $A R$, and on the medical topics. The AR topics covered in the course are similar to those in the typical design and media programs and include volumetric

\footnotetext{
${ }^{13}$ http://34.248.116.126/media-v8/aqeo/modules/2020-21/GAV5023-B_AugmentedReality-Design,-Principles-and-Practice_MD202021.pdf

${ }^{14}$ https://web.archive.org/web/20200913183132/http://campar.in.tum.de/Chair/ TeachingWs19MedAR

${ }^{15}$ https://web.archive.org/web/20200914082259/https://camp.lcsr.jhu.edu/ spring2019/

${ }^{16}$ https://web.archive.org/web/20200913183344/https://www.gla.ac.uk/postgraduate/ taught/medicalvisualisation
}

visualization, 3D modeling, interactive application development, and AR/VR development.

The Augmented Reality \& Smart Objects course at the Polytechnic University of Catalonia is an example of teaching the topics of AR together with sensors and wearables as part of an engineering study program ${ }^{17}$. The course focuses on mobile and location-based AR development and sensors and IoT. The practical assignments are done in Unity and Arduino.

AR courses also appear in the study programs in humanities, social sciences and business. Such courses usually focus on the application of AR in the relevant domains and using AR authoring tools. For example, the School of Humanities and Digital Sciences at the Tilburg University offers a series of courses that approach AR from the humanities and social science perspective ${ }^{18}$. These courses cover the basic concepts of XR, the value for the humanities and social sciences, creating basic XR experiences using authoring tools, assessing XR for applications, ethical and accessibility issues, and communicating XR topics.

Another example is the Virtual and Augmented Reality Master's program offered by the Department of Art, Design \& Computer Science at the York St John University ${ }^{19}$. The program integrates the AR with its application to the marketing domain. The AR topics covered by the program are similar to those in the typical CS programs with the focus on HCI. The topics include design thinking and design research methodologies, XR application and project design, and AR/VR development in the form of a major project. The programme also contains courses on marketing communications and brand management.

There are few multidisciplinary curricula that integrate AR with its application domains. Among them, only the area of medical AR is a recurrent example, also including cross-institutional collaboration. The shortcoming of such courses is that they are driven by the application domains, including different sets of AR skills ranging from the introduction of basic concepts and using authoring tools to the hardware and visualization skills. On the other end of the spectrum, they often remain tied in to low level processing, as catering for specialist visualisation imposes non-standard requirements.

\subsection{Multiple Courses}

There are only a few examples of universities splitting the content of an AR curriculum across multiple courses, not counting specialised study programmes. TU Wien offers three complementary courses on AR in the Faculty of Computer Science. The fundamental theoretical course $\mathrm{e}^{20}$ includes introduction, industry applications, hardware and delivery systems, 3D graphics, 3D interaction, and psychological aspects (2 ECTS / 1 US / 4 UK points). The practical course ${ }^{21}$ includes introductory modular tasks and tutorials to

\footnotetext{
${ }^{17}$ https://web.archive.org/web/20200913183422/https://www.upc.edu/en/masters/ applied-telecommunications-and-engineering-management-masteam

${ }^{18}$ https://web.archive.org/web/20200913183524/https://catalogus.tilburguniversity. edu/osiris_student_tiuprd/OnderwijsCatalogusZoekCursus.do

${ }^{19}$ https://web.archive.org/web/20200913183603/https://www.yorksj.ac.uk/courses/ postgraduate/arts-humanities--performance/virtual-and-augmented-reality-ma/ ${ }^{20}$ https://web.archive.org/web/20200913183642/https://tiss.tuwien.ac.at/course/ courseDetails. $x$ html dswid $=2676 \&$ dsrid $=977 \&$ courseNr $=188369 \&$ semester $=2019$

${ }^{21}$ https://web.archive.org/web/20200913183750/https://tiss.tuwien.ac.at/course/ courseDetails. $x$ html ?dswid $=2935 \&$ dsrid $=903 \&$ courseNr$=188913 \&$ semester $=2019 \mathrm{~W}$
} 
complement the theoretical knowledge as well as a project assignment to develop an AR app (4 ECTS/ 2 US / 8 UK points). The third course $^{22}$ aims to cover more advanced topics, such as hardware, tracking and object segmentation based on depth data, integration of sensor data, perception, rendering in AR, and haptics (3 ECTS / 1.5 US / 6 UK points).

Splitting the AR curriculum can also be done by creating a core and an optional course. For example, the master's in $\mathrm{HCI}^{23}$ at the University of Nottingham includes the core course Mixed Reality Technologies that covers design and development of cross reality (XR) applications, IoT and sensors, specifically focusing on the user interaction. The complementary elective course Simulation, Virtual Reality and Advanced Human-Machine Interface adds such topics as multimodal interfaces, natural language and gesture interfaces, accessibility, and more.

At the same time, there are practices of multiple courses on AR existing at the same university, but offered by different departments and not complementing each other.

For example, the CS department of the National University of Singapore offers a course Interaction Design for Virtual and Augmented Reality ${ }^{24}$ that covers topics such as an overview of XR, perception, XR interaction, speech and gesture interactions, tracking, computer vision, collaboration in XR, health issues, and industry perspectives. At the same time, the Department of Communications and New Media at this university offers the described above Mobile Interaction Design course ${ }^{25}$ that covers design and usability for mobile AR.

At the Liverpool John Moores University, two separate Masters programs are offered on AR-related topics. First, a CS Master's program with a specialization on Virtual and Augmented Reality ${ }^{26}$ covers software engineering for XR, shared spaces, UX for XR, perception, hardware, visual fidelity, real-time rendering. Second, a Master's degree in Immersive Arts ${ }^{27}$ focuses on immersive storytelling, XR prototyping, practice-based and theoretical research methods, enhanced by collaborative project work and placements in a creative sector.

Complementing courses, such as at TU Wien and University of Nottingham, are useful in structuring the material. At the same time, only a few existing examples and the different ways of splitting practices add an extra layer of complexity for students and teachers trying to understand how the courses fit together. Additionally, the mono-disciplinary offers by different departments may work well within the discipline, but can be confusing for industry, providing different skills models for recruiters.

\footnotetext{
${ }^{22}$ https://web.archive.org/web/20200913183852/https://tiss.tuwien.ac.at/course/ courseDetails . $x$ html dswid $=6266 \&$ dsrid $=604 \&$ courseNr $=188456 \&$ semester $=2020 \mathrm{~S}$ ${ }^{23}$ https://web.archive.org/web/20200913183932/https://www.nottingham.ac.uk/ pgstudy/course/taught/human-computer-interaction-msc

${ }^{24}$ https://web.archive.org/web/20200913184003/https://ivle.nus.edu.sg/V1/lms/ public/view_moduleoutline.aspx?CourseID=06C4D3A7-D2FF-4A67-A7C56E9EC32D910B

${ }^{25}$ https://web.archive.org/web/20200913184046/https://ivle.nus.edu.sg/V1/ $\mathrm{lms} /$ public/view_moduleoutline.aspx?CourseID=73cc931e-e316-49b7-8dc012f05b31042d

${ }^{26}$ https://web.archive.org/web/20200913184119/https://www.ljmu.ac.uk/study/ courses/postgraduates/computer-science-virtual-and-augmented-reality

${ }^{27}$ https://web.archive.org/web/20200913184212/https://www.ljmu.ac.uk/study/ courses/postgraduates/immersive-arts
}

\subsection{Specializations}

$\mathrm{AR}$ and related topics are not only included into mono- or crossdisciplinary study programs as courses, but also create the basis for specialized study programs and specializations.

A prominent example of such programs is the master's program in CS with a specialization in Augmented \& Virtual Reality at Trinity College Dublin ${ }^{28}$. In addition to the fundamental CS modules, shared by all specializations, it includes specific modules on graphics and vision in the courses Computer Vision, Computer Graphics, Mathematics of Light and Sound, Real-time Rendering and Realtime Animation. The AR course in this program (as presented above) extends the introductory computer vision course and covers more advanced technical AR topics through tutorials, practical assignments and a final project. The program focuses solely on technical aspects, meaning that topics such as creative design, storytelling and social aspects are largely missing.

The previously mentioned specialization on Virtual and Augmented Reality ${ }^{29}$, which is part of the CS program at Liverpool John Moores University uses a different approach. It has a stronger focus on software engineering for XR, AR/VE development and SDKs, UX design for XR, geometry and algebra, digital content and scene design, collaborative XR, and perception. At the introductory level, the program covers hardware, graphics and rendering, but does not include computer vision and AI.

The Virtual Reality and Smart Technologies program ${ }^{30}$ at the Vidzeme University of Applied Sciences is not a specialization of a CS program, but largely focuses on the same topics, as the two examples above. It contains modules on AR and VR design and development, UX, content authoring, as well as advanced graphics and AR topics such as geometry processing, computer vision, and machine learning.

Another notable example is a masters in Computer Vision ${ }^{31}$ offered by the Mohamed bin Zayed University of Artificial Intelligence that covers many AR-related topics, such as 3D Computer Vision, Graphics, and AI. At the same time, this program does not cover either AR or VR development, and only mentions AR as a possible application area of the topics covered in the program.

A different example of specialized programs is the two-year program Augmented Reality - Augmented and Virtual Reality offered by the Digital Media Department of the Ballyfermot College of Further Education ${ }^{32}$. The program focuses on the art and media aspects covering an introduction to XR as well as the theory and practical projects in motion graphics, visual effects, 3D modeling, animation, audio technologies, and XR scene design. The program does not include any CS topics.

A notable exception from this pattern is the Virtual and Augmented Reality program at the Goldsmiths University of London ${ }^{33}$.

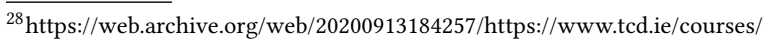
postgraduate/az/course.php?id=DPTCS-AVRE-1F09

${ }^{29}$ https://web.archive.org/web/20200913184119/https://www.ljmu.ac.uk/study/ courses/postgraduates/computer-science-virtual-and-augmented-reality

${ }^{30}$ https://web.archive.org/web/20200913184450/https://va.lv/en/study-here/mastersdegree/virtual-reality-and-smart-technologies/programme-content

${ }^{31}$ https://web.archive.org/web/20200913184528/https://mbzuai.ac.ae/study/ academic-programs/msc/computer-vision

${ }^{32}$ https://web.archive.org/web/20200913184638/https://www.bcfe.ie/courses/ augmented-reality-augmented-virtual-reality-arvr-hnd/

${ }^{33}$ https://web.archive.org/web/20200913184958/https://www.gold.ac.uk/pg/ma-mscvirtual-augmented-reality/
} 
The program approaches AR form the game-design perspective and mostly combines AR/VR development topics with design and media topics. It contains a small number of core courses covering the introduction to XR, programming for games, 3D modeling and animation, math, and graphics. In addition, the program contains a large number of optional courses, most of which are multidisciplinary combining both CS and design aspects. The optional courses include advanced programming for games, interactive graphics, game design, game analytics, AI for games, data and machine learning for artistic practice, and creative coding. The program also offers some monodisciplinary design courses, such as animation, 3D modeling, and special effects.

Most specialized programmes we reviewed here approach AR from a mono-disciplinary perspective, usually tied in to the departments where they are offered. One or two-year programmes have the space to include a variety of courses, but currently most of them provide deep knowledge and skills within a single discipline. This approach does not fully satisfy industry needs for specialists with a wide range of both technical and creative skills, as identified in section 4.1 .

\subsection{Online Offers}

A variety of courses focusing specifically on AR are also available on online platforms. Compared to training on other emerging topics in computer science, such as VR and AI, courses on AR remain relatively limited. Similar to their academic counterparts, online courses on the topic of AR are typically taught in conjunction with VR. The vast majority of such courses are short, lasting several hours in duration and focus on only one particular aspect of AR application design. They can therefore be classified as tutorials. To conduct a systematic search of relevant courses, the following terms were used: "Augmented Reality", "Mixed Reality", "Extended Reality", "Computer vision", "Spatial computing", ARKit, "AR Core", Hololens. Given the interest of the current study in assessing comprehensive AR curricula, the list of results was further refined using exclusion criteria. These removed short tutorials to arrive at a list of courses whose contents matched the depth and specificity of university programs. The following parameters were set to arrive at the final selection:

- Courses with at least $30 \%$ of the content dedicated to AR

- Courses equivalent to at least 30 study hours

- Courses whose curriculum was freely available online

A search on Massive Open Online Courses (MOOC) platforms identified a single semester-long online course focusing on AR comparable to those delivered within the physical university setting. Titled, 'Work with augmented reality (AR) and the web', the training is provided by Curtin University on the platform edX ${ }^{34}$. It forms part of a Professional Certificate in the Department of Computer Science and spans eight weeks, requiring an investment of 8-10 hours/week. The content focuses on the creation of AR apps using Xcode and the Swift programming language. It covers topics such as user interaction, input and system controls, app design, $\mathrm{HCI}$, closures, animation, and issues of communication with the web and concurrency.

\footnotetext{
${ }^{34}$ https://web.archive.org/web/20200913185035/https://www.edx.org/course/workwith-augmented-reality-ar-and-the-web
}

A similar but shorter offering, 'Getting started with Augmented Reality' is a course offered by Institut Mines-Télécom on Cours$\mathrm{era}^{35}$. The course offers 19 hours of content (below our exclusion criteria) and contains four modules, covering the introduction of AR concepts, including typical architectures of AR apps, the ARAF format $^{36}$, AR SDKs and location-based AR. The course offers three practical exercises developing mobile AR applications using ARAF.

\subsection{Commercial Offerings}

A wide number of courses and tutorials on AR can also be found on commercial websites. Searches for AR on Udemy, a teaching and learning marketplace, returns 60 results, of which only three are longer courses devoted specifically to AR. Two of them titled 'Artificial Intelligence \& Augmented Reality in Unity \& Xcode'37 and 'Unity \& AR: Make a Unity Game \& an AR App for iOS'38 offer a set of overlapping material. Both are designed for beginners and last 20.5 and 27.5 hours respectively, somewhat shorter than our exclusion criteria of 30 study hours. They offer a broad survey of different approaches to AR application development, including an introduction to Unity, Xcode, and Swift.

The lengthiest course identified on a commercial platform was the Udemy 'Learn 3D AR technology with Xcode and Python!', which is aimed at beginners and offers 100.5 hours of instruction ${ }^{39}$. Relying on more than 100 videos, 327 lectures, 51 articles and 43 downloadable resources, the training takes a coding approach to AR development. It does so by introducing coding in Xcode, Python, HTML, CSS, C\#, and R. The focus rests mainly on providing an introductory understanding of these programming languages and on information visualisation, rather than on delving deeply into AR development.

The Interaction Design Foundation offers an 18 hour course How to Design for Augmented and Virtual Reality ${ }^{40}$. The course is offered by an online provider that focuses specifically on design education. It covers both theoretical and practical aspects of the HIC perspective on AR, including the topics of storytelling, UX and spatial UI, and multimodal interaction and designing content, such as $3 \mathrm{D}$ characters.

Microsoft, the maker of HoloLens, also offers a number of courses in $\mathrm{AR}^{41}$. These can be classified as tutorials, each being dedicated to a specific aspect of working with the HoloLens, such as instruction on gesture, gaze, voice, motion controllers, spatial sound, or spatial mapping. Introductory training on game engines such as 'Getting started with Unity' or the Unreal engine is also the focus of other modules. Although alone these courses can be categorized as tutorials, together they can be seen as forming a curriculum

\footnotetext{
${ }^{35}$ https://web.archive.org/web/20200913185108/https://www.coursera.org/learn/ augmented-reality

${ }^{36}$ https://web.archive.org/web/20200913185154/https://mpeg.chiariglione.org/ standards/mpeg-a/augmented-reality-application-format

${ }^{37}$ https://web.archive.org/web/20200913185230/https://www.udemy.com/course/ artificial-intelligence-augmented-reality-in-unity-xcode/

${ }^{38}$ https://web.archive.org/web/20200913185423/https://www.udemy.com/course/ unityaugmentedreality/

${ }^{39}$ https://web.archive.org/web/20200913185504/https://www.udemy.com/course/ learn-3d-ar-technology-with-xcode-and-python/

${ }^{40}$ https://web.archive.org/web/20200913185552/https://www.interactiondesign.org/courses/how-to-design-for-augmented-and-virtual-reality

${ }^{41}$ https://web.archive.org/web/20200913185624/https://docs.microsoft.com/enus/windows/mixed-reality/tutorials
} 
of sorts. The focus rests heavily on the practicalities of working with the technology, rather than the lasting knowledge of the field, which renders these tutorials particularly susceptible to becoming outdated as technology improves.

Other producers of AR software or hardware likewise feature tutorials on their websites. PTC, the solutions provider and developer of the Vuforia AR SDK stands out ${ }^{42}$ for also providing complementary information that showcases the potential of AR applications in industry ${ }^{43}$. In doing so, it highlights a valuable approach, that of designing niche courses focused on the practical applications of the technology in particular sectors, which is an area of opportunity for many other fields.

Professional communities and networks are also becoming engaged in teaching AR. VR First in cooperation with the AR industry has built an XR curriculum to be taught in a coding bootcamp format $^{44}$. The program includes an introduction to XR and three sets of practical electives on AR/VR development (Unity, programming, agile development, version control), real-time 3D art (basics of computer graphics, 3D modeling and animation), and UI/UX and game design (interaction design, storytelling, UX and UI, game design, UX evaluation, and optimization). The program also has a strong focus on the industrial cases, workflows and production.

It is clear that despite a wealth of short tutorials, the offering of comprehensive AR courses online remains limited, and within this selection no clear curricular trend emerges. Although a highlight is that little to no background knowledge is needed to enroll, such courses remain fairly conservative in their design, are narrowly focused and not particularly innovative in the topics they cover or curriculum. There remains a significant gap in the market for comprehensive online training courses on AR.

\subsection{Industry-Academia Partnerships}

Targeted training and recruiting is a significant challenge (and cost factor) for the AR industry, easily amounting to recruitment costs of $12-22 \%$ of an annual salary ${ }^{45}$ for a single appointment. Partnerships between industry and academia can be mutually very beneficial, offering graduates a perspective and companies access. Several examples, listed below, stand out.

The Technical University of Munich, together with several universities and hospitals have partnered with IT and AR companies to deliver Medical AR Summer School ${ }^{46}$. The focus of this program is to teach new generations of scientists recent advances in Augmented Reality as well as to strengthen the connection between medicine, science and industry. The course includes keynotes from leading scientists in the field, insights from medics and technical expertise, along with the theoretical applications of 'Medical Augmented Reality'. The training also enabled participants to create their own Medical AR solutions in interdisciplinary teams by using cutting-edge technologies.

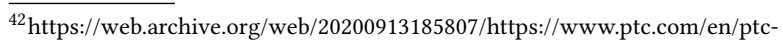
university

${ }^{43}$ https://web.archive.org/web/20200913185859/https://www.ptc.com/en/resources/ augmented-reality/white-paper/getting-started-with-industrial-ar-solutions

${ }^{44}$ https://web.archive.org/web/20200913185926/https://xrbootcamp.com/

${ }^{45}$ https://web.archive.org/web/20200913190056/https://www.quarsh.com/blog/thetrue-cost-of-recruitment/

${ }^{46}$ https://web.archive.org/web/20200913190218/https://www.

medicalaugmentedreality.org/
}

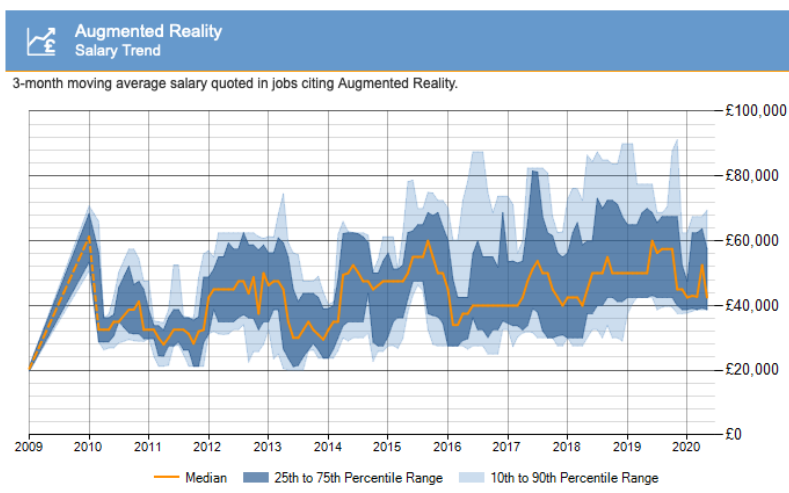

Figure 1: Three-month moving average salary quoted in jobs citing 'Augmented Reality' (Source: ITJobsWatch).

The Performance Augmentation Lab of Oxford Brookes University in collaboration with the industry partner, DAQRI, organised The AR Winter School ${ }^{47}$. The week-long training program, spanning 8-8.5 hours/day, included 17 hours of lectures, a 1.5 hour introduction to $\mathrm{AR}$, an overview of ARToolKit, and several practical ARToolKit workshops, including training on JSARToolKit and Three.js. Lectures and workshops also focused on using Unity3D to develop AR applications, interaction design for AR applications and training on advanced AR technologies. The lectures and practicals were complemented by an equal amount of time spent on group work building AR applications. An evening visit to an AR arts installation (The Reading Machine) complemented the program.

The University of Auckland partnered with Magic Leap to deliver the Augmented Reality Summer School ${ }^{48}$. The week-long course included 12 hours of lectures and 30 hours of hands-on development with the Magic Leap smart glasses. The lectures covered the introduction to AR, UX design for Magic Leap display, developing Magic Leap applications with Unity, rapid prototyping for AR, AR interaction, AR content development, perception, designing collaborative systems, AR best practices, evaluating AR systems, and future research in AR. Attendees broke up into small groups to complete prototype Magic Leap applications that were presented at an open event at the end of the course. Magic Leap supported the event by loaning their smart glasses and delivering guest lectures.

These examples show that short but focused training delivered by academic institutions in collaboration with commercial partners, whose design combines instruction and project work, is a successful way of instilling new AR skills. Such courses also attract students from a variety of disciplines and so are a good way to break disciplinary barriers and to upskill. The major shortcomings of such partnership courses are the binding to specific development frameworks and time restrictions that usually limit practice in tutorials and small development projects. Moreover, as collaboration is often connected to specific recruiting needs of industry, which typically remain niche.

\footnotetext{
${ }^{47}$ https://web.archive.org/web/20200915192841/https://pal.cct.brookes.ac.uk/ augmented-reality-winter-school-2017/

${ }^{48}$ https://web.archive.org/web/20200913190602/http://empathiccomputing.org/ magicleap-workshop/
} 


\subsection{Summary of Prevalent Shortcomings}

Our survey has found a wide range of AR training offers of varying quality both in and outside of CS programs. AR today is taught at all levels and as part of a variety of disciplines and, in consequence, industry does not know what skills to expect graduates to possess.

We found that even the same university may offer different courses in different departments, which confuses both students and potential employers. As employers do not know the pools from which to recruit, they needlessly multiplying their efforts.

Course offerings are often mono-disciplinary, focusing on HCI aspects, information systems aspects, software engineering aspects, communications degree, design degree, but rarely a combination of these approaches. This is true not only for the CS programs, but also for AR courses or related topics taught as part of design/media/communication programs. This challenge is perhaps similar to other 'hybrid' areas that require both creative and computing skills, such as games development, but for AR this challenge has not yet been addressed. This prevents the education of specialists with a broad range of AR skills (AR generalists), requiring industry to employ teams of specialists, which hinders in particular microenterprises and SMEs in the uptake of AR. A model-T approach would be more suitable, with a foundations course providing broad overview, and an advanced course offering further deep specialisation.

As time advances, AR has become more high-level, with abstractions being introduced. Low level libraries like the OpenCV computer vision library are no longer needed on the undergraduate level, as consumer-facing apps do not require knowledge on that level anymore. Such knowledge is only needed for advanced AR such as for medical imaging. The educational offers lag behind these changes. It is unclear what type of training is needed and at what level.

Industry-driven programs are too short-sighted, not covering foundations to guide and help integrate future knowledge. Most of the commercial offers are too close to technology, only looking at particular tools, already dated when published. Short practical tutorials on using specific tools dominate over the lasting knowledge of principles.

We identified a lack of a commonly accepted skills framework or expected skills portfolio for graduates. Students do not develop the reference frame needed to autonomously lead the continuation of their education through independent study.

Curriculum material is often not aligned to industry needs. Students in AR courses may not be trained on current software tools and hardware, or may not get the skills needed by industry (e.g., lack of understanding of Computer Vision). There is no reference model. Graduates can be anything or nothing.

There are very different assessment models, ranging from small assignments to project work to exams, with varying weightings as to how important combinations of these are. We cannot distinguish a pattern. Assessment seems to largely depend on the context of the program or school in which the course takes place. Nonetheless, there are no universally accepted assessment schemes, nor is there a comprehensive mapping of what the assessment options and dependencies to the course organisation options are.
There is no agreed curriculum in the professional societies, and therefore no foundation for independent auditing and accreditation. Third party audit through professional societies is impossible.

There is no institutional body or expert commission that revisits the model curriculum to assess whether it is still up to date. The curriculum may vary significantly from institution to institution.

There is a lack of AR Standards [13]. Practical exercises are often bound to a single platform. Cross-platform and cross-device is still an issue. This increases the workload of lecturers in preparing and teaching, while single framework training poses a barrier to adoption. Many resulting applications remain proof-of-concept.

Despite ongoing debates on the ethical implications of AR use [10], these topics are rarely addressed in the curricula we identified. Considering how AR intersects with aspects of private and public space, ethical issues should be addressed in the context of developing AR applications and hardware. Salient examples of ethical dilemmas surrounding this topic include the appearance of PokemanGo at Auschwitz [15], the intersection of AR with facial recognition [31] and the asymmetry of equitable access to AR (its appeal in underprivileged communities, or the cost of hardware) and the way in which this might reinforce already existing stratification of society.

\section{MODEL AUGMENTED REALITY CURRICULUM}

We organise our proposal for a model AR curriculum along four dimensions: skills, lesson plan, course format, and assessment regime.

We consider the list of skills provided in Section 4.1 as an inventory of skills the job market is looking for, a framework for skills development focused only on the 'hard', technical skills. We deliberately excluded from this list the rich professional skills required of any applied CS area (like project management skills). These will have to be complemented when writing an actual syllabus and we make some recommendations on that in the sections on intended learning outcomes for both blueprints.

In Section 4.2 we present the lesson plans in the form of a list of possible units of lectures and tutorials for both a Foundations as well as an Advanced course module blueprint. We deem few lectures and tutorials to be 'must', some 'should', and others 'supplementary' so as to maximise flexibility in bootstrapping an actual course from these blueprints. We recommend intended learning outcomes for each course module, and analyse their profile regarding the skills framework introduced.

Section 4.3 describes a course format and options for how the lectures and tutorials can be stacked and what leeway exists. Typically, the module size already prescribes the effort students need to put in regarding their learning work. If you aim for a module of a certain size, you have to bring the hours. For example, 7.5 ECTS in Europe (about 4 US points or 15 UK points) are about 200 hours of learning work, so whatever the exact number of contact hours from lectures and tutorials, there will be a significant share of independent project work and preparation for assessments.

Last, but not least, in Section 4.4 we describe the key rationale for the assessment regime and portray different options we can recommend regarding the actual marking scheme. 


\begin{tabular}{|c|c|c|}
\hline No & Group & Specific skills \\
\hline 1 & $\begin{array}{l}\text { General } \\
\text { AR Development Skills }\end{array}$ & $\begin{array}{l}\cdot \text { AR SDKs (ARcore, ARkit, MRTK, WebXR, etc.) } \\
\cdot \text { VR SDKs (PSVR, Oculus, OpenVR, WebVR, etc.) } \\
\cdot \text { Unity } \\
\text { · Unreal Engine }\end{array}$ \\
\hline 2 & $\begin{array}{l}\text { Platform-specific } \\
\text { Development Skills }\end{array}$ & $\begin{array}{l}\cdot \text { iOS (Objective C, Swift, Xcode, etc.) } \\
\cdot \text { Android (Java, Kotlin, Android Studio, etc.) } \\
\cdot \text { Linux, Windows, MacOS } \\
\cdot \text { Web as a platform } \\
\cdot \text { Multi-platform, cross-device } \\
\cdot \text { Embedded systems and real-time embedded systems } \\
\cdot \text { Cloud and Edge Computing } \\
\cdot \text { Scaling (Modules, Microservices, etc.) }\end{array}$ \\
\hline 3 & $\begin{array}{l}\text { Specific Programming } \\
\text { / Markup Language } \\
\text { Skills }\end{array}$ & $\begin{array}{l}\text { - Object Oriented Programming ( .NET, C\#, Mono, Java, C++) } \\
\cdot \text { Procedural Programming (C, Ruby, Python) } \\
\cdot \text { JavaScript/HTML (three.js, AR.js, A-frames) }\end{array}$ \\
\hline 4 & $\begin{array}{l}\text { Advanced } \\
\text { Computing } \\
\text { Skills }\end{array}$ & $\begin{array}{l}\text { - GPU programming for advanced shading (such as NPR, global illumination, shadow, particles) } \\
\text { - Hardware accelerated computation (such as Neon, SSE, GPGPU, DSP...) } \\
\text { - CPU and GPU optimisation } \\
\text { - Graphic APIs (OpenGL, OpenGL ES, Vulkan or DirectX) } \\
\text { - Compute APIs (CUDA, OpenCL, etc.) } \\
\text { - Software optimisation and instrumentation } \\
\text { - Multi-threaded programming }\end{array}$ \\
\hline 5 & $\begin{array}{l}\text { Computer Vision } \\
\text { Skills }\end{array}$ & $\begin{array}{l}\text { Computer vision, image analysis, image processing, OpenCV } \\
\text { - Object detection, recognition, pose tracking (Vuforia, MaxST, ARToolKit, Wikitude, SolAR, etc.) } \\
\text { - Spatial mapping and understanding }\end{array}$ \\
\hline 6 & $\begin{array}{l}\text { Computer Graphics } \\
\text { Skills }\end{array}$ & $\begin{array}{l}\text { 3D modelling (3ds Max, Maya, Modo, Blender, etc.) } \\
\text { - Interactive 3D graphics design } \\
\text { - Rendering (Vray, Corona, Octane, etc.) } \\
\text { - CAD tools (Autocad, Solidworks, Catia, Geometric dimensioning / tolerancing, etc.) } \\
\text { - Generic CG \& VFX (Texturing, Animation, 3D modelling, lighting \& rendering) } \\
\text {-3D math and Math for graphics }\end{array}$ \\
\hline 7 & $\begin{array}{l}\text { Advanced Data } \\
\text { Analysis and AI Skills }\end{array}$ & $\begin{array}{l}\text { - Data analysis and manipulation (Matlab, R, SPSS, etc.) } \\
\cdot \text { Machine learning, deep learning libraries (TensorFlow, Torch, Caffe, etc.) } \\
\cdot \text { Artificial Intelligence (Watson, LUIS, etc.) } \\
\cdot \text { Big data and data mining } \\
\text { - Algorithms }\end{array}$ \\
\hline 8 & $\begin{array}{l}\text { Audio Engineering } \\
\text { Skills }\end{array}$ & $\begin{array}{l}\text { Spatial Audio (Audio DSP, FMOD) } \\
\text { - Sound design, Composition and Production (sound generation, mixing, etc.) }\end{array}$ \\
\hline 9 & $\begin{array}{l}\text { Interaction Technology } \\
\text { Skills }\end{array}$ & $\begin{array}{l}\text { - Gesture interaction } \\
\text { - Eye Gaze tracking/interaction } \\
\text { - Speech interaction } \\
\text { - Accessibility }\end{array}$ \\
\hline 10 & $\begin{array}{l}\text { Design and Rapid } \\
\text { Prototyping Skills }\end{array}$ & $\begin{array}{l}\text { - User interface design and storytelling } \\
\text { - Basic artistic/design skills (hand sketching, illustration, etc.) } \\
\text { - Design tools (Adobe Creative Suite, InVision, Sketch, whiteboards, paper sketches, Adobe XD or Illustrator) } \\
\text { - User-centred design (Accessibility and Universal Design, UX design, Usability, etc.) } \\
\text { - Authoring tools (Wikitude, InVision, Adobe Aero, etc.) }\end{array}$ \\
\hline 11 & $\begin{array}{l}\text { Hardware and } \\
\text { Hardware-related } \\
\text { Skills }\end{array}$ & $\begin{array}{l}\cdot \text { AR Smart Glasses (HoloLens, Magic Leap, Epson BT series, etc.) } \\
\cdot \text { AR projection solutions } \\
\cdot \text { Optical design (Optical design software, optics, and frames) } \\
\cdot \text { Maker skills (3D printing, arduino, microcomputers) } \\
\cdot \text { Robots } \\
\cdot \text { Internet of Things } \\
\cdot \text { Sensors } \\
\cdot \text { Wearables } \\
\cdot \text { Signal processing (for context sensing or for sensor fusion) } \\
\cdot \text { Drivers, Hardware Abstraction Layer }\end{array}$ \\
\hline 12 & User Evaluation Skills & $\begin{array}{l}\text { Types of User Evaluation (informal, formal, heuristic, etc) } \\
\text { - Design of User Experiments } \\
\cdot \text { Methods for Experiment Data Analysis (SPSS, R, etc) } \\
\text { - Reporting on user studies } \\
\text { - Accessibility }\end{array}$ \\
\hline
\end{tabular}

Table 1: Augmented Reality skills framework (refined from [16]) 


\section{1 'Hard' Skills}

From a comprehensive job market study conducted in [16], an initial table of skills was extracted and now further refined with the expert input from the curriculum working group. We have excluded, though, more generic software development skills and project management skills, as both are not specific to the specialisation in AR, but rather generic. Deliberations on how to integrate these in lesson plans and learning objectives can be found in the according sections below. Amongst the 'hard' skills for AR, we identify 12 groups of skills listed in Table 1.

One can notice the variety of the 'hard' skills for AR we identified. This may lead to formulating statements like "creating meaningful AR applications can not be done by individuals alone but they should team up, therefore aggregating disciplinary expertise". Such a claim is not specific to AR. For example, many of the modern successful games are created by interdisciplinary teams. There are few successful games, however, created by individuals alone.

Of the 12 groups of skills, 11 groups (from 2 to 12) are not specific to AR, but can be used for other domains. This can be interpreted as a positive fact. Potentially, it would be possible to 'only' train someone with AR development skills from group 1 if the person already has some or all of the more traditional skills from groups 2 to 12 . Naturally, people with expertise in groups 2, 3, and 4 would have less difficulties to learn AR-specific skills of group 1.

A particular situation occurs for skills in groups 5, 6, 7, 8. These are technical skills needed to understand how specific components (e.g., object trackers and rendering engines) of AR systems work. In order to use these components, however, a superficial comprehension is enough. Indeed, such components are well encapsulated in dedicated libraries that are exposing and documenting only a small set of interfaces. Less is the case for groups 9 and 10. These skills are affecting the conceptual phase of AR applications and not mastering them may affect the impact and utility of the end result.

Skills in group 10 should help the AR application designer (or team) to understand the limitations and constraints imposed by the hardware and find appropriate solutions to avoid or compensate them. The ultimate AR device allowing for perfect alignment between real and virtual is not available today and it may not be available in the next few years. Therefore, having a deep understanding on how they work and what they are able to do and not is a key element for creating meaningful AR experiences.

Finally, skills in group 12, referring to the need of conducting user-centered design, should take into consideration the multimodal and multisensory aspects of AR. They refer not only to the reaction of the user to digital content (as for more traditional cases of video and audio) but also to the reaction of the user to how sharp is the border between the perception of the natural artefacts and the digital ones.

\subsection{Lesson Plans}

The suggested curriculum is broken units of lectures and practical tutorials. Lectures in general are conceptualised to cover theory and foundations, while the tutorials are expected to complement them with selected practicals. For example, a lecture on evaluation would cover a broad overview on AR-relevant HCI methodologies and all relevant detail for key methods, whereas the practical tutorial would pick one or two key methods and practice their application from administering the questionnaire to data analysis and representation.

In the provided recommendation, we also divide all units into three types used in the ACM curricula recommendations: must, should, and supplementary. The core units cover topics and involve practices that all students must learn and experience. These units are marked with $[\cdots . . \cdot$ ] next to their titles. Another part of units cover topics and include experiences that the students should have. These units are marked with [••]. All other units cover supplementary or optional material, and the actual selection can be modified based on target audience and local needs. These units are marked with [•]. For example, an AR course taught in CS might have a lot of technical programming optional content, while in a Design School there might be more content about storytelling, rapid prototyping, and user experience. In our recommendations, the units marked with [.... $]$ may include topics that are taught in other courses in the study program. For example, a tutorial on AR Engine introduction is a must for the AR course, but it might be covered in a game-design course as well.

The advanced course builds on the foundations course. Depending on local programs, the foundations can be included in an undergraduate degree and the advanced in a master's course. It is certainly also possible, however, to include both in a master program. Until there is more foundational work already in secondary school or college, however, it is unlikely that both can be taught on a broader scale at bachelor level, no matter how targeted the selection of students.

4.2.1 Lesson Plan for Foundations. This course presents an introduction to AR, with emphasis on designing and developing AR applications. The course starts with a comprehensive introduction to the field, covering also its history with early precursors dating back to the 19th century and with more than half a century of serious technology R\&D. The course then introduces the state of the art of hardware and software, with practical try-out possibilities of Smart Glasses, interactive clothing, and other futuristic technology. The course covers all necessary material about Spatial Computing, Human Computer Interaction, Perception, Design Thinking, and Application Development. A rich mix of theory and practice is complemented with methodology and hands-on development and evaluation. Insights into specialist application areas and job perspectives help sharpen the students' skills set.

Lecture $1[\cdots \cdot \cdots]$ Introduction to AR. This lecture guides through the history of AR (starting 400 years ago and till modern software and hardware platforms), covers the key concepts in AR, introduces augmentation pipeline, explaining new ideas of "Experience" and state of the art in AR technologies.

Lecture 2 [•.••] Human Perception and Processing. This lecture introduces the foundations of research and design methodologies in $\mathrm{HCI}$, the Human Perception requirements for AR, and why different methods should be used for designing AR experiences.

Lecture $3[\cdots \cdots]$ Technology Overview. This lecture covers the understanding and evaluation of technology alternatives from enduser development to high- and low-level software engineering. It presents the principles of assessment if AR is the right technology for a specific purpose, which combination of AR display, tracking 
and interaction technologies are suitable. It develops a deeper understanding of component-level technology, such as display hardware and introduces available sensor hardware, interaction technology, and delivery systems. The lecture also briefly covers rapid prototyping in AR.

Lecture $4[\bullet \cdot$ Experience Engineering. This lecture covers details on Design Thinking, the importance of context, user-oriented design, best practices in experience design and examples of "how not to".

Lecture $5[\bullet \cdot$ HCI evaluation methodologies. It covers experiment design and evaluation methodologies like technology acceptance and use, system usability, user satisfaction with hardware and interaction itself.

Lecture $6[\cdots \cdot \bullet]$ Spatial Computing. In this lecture, we introduce the concept of Spatial Computing and provide several best and worst practices of AR designs, illustrating them by examples of solutions and applications.

Lecture $7[\bullet]$ Software Development Methods. The lecture covers agile software engineering models versus traditional methods. It explains scrum processes and kanban in detail. Moreover, agile practices like code style guides (definition of done), source code reviews after sprints, source code management (git) and DevOps basics are addressed.

Lecture $8[\cdot]$ Geometric Algebra. Reviews the mathematics needed for AR and computer graphics, such as tools that model rotations, understanding of the geometric interpretation of complex numbers, and pros and cons of using matrices, complex numbers, Euler angles or quaternions.

Lecture $9[\cdot]$ Storytelling. The lecture covers the basic principles in storytelling, presents methods such as storyboarding, and provides tips and tricks on how to engineer an experience.

Lecture 10 [ $\bullet$ Careers in AR. This lecture gives an overview of the job market, what employers want, and the skills framework that helps to keep track of developing the sought-after talents.

Lecture 11 [ $\cdot$ ] Design Inspiration. Typically provided by local industry, this talk introduces to real client projects, highlighting how they were developed and designed.

Lecture $12[\bullet \cdot$ Research Directions. This lecture needs constant updating, incorporating new Grand Challenges and latest achievements, as for example available from the IEEE ISMAR conference annual summaries and highlights. At the time of writing, this lecture provides an outlook on future developments and novel tools such as consumer-grade volumetric capture, hearables, voice-activated graphics, or brain-computer interfaces and innovative application areas such as Digital Assistants, Diminished reality, Deep Fakes, Programmable Synthetic Hallucinations, Umwelt Hacking, Perceptual Adaption, Sensory Augmentation, Neuro Design, or Wearable Skin. The lecture also covers the ethics of AR and the social implications of the technology.

Tutorial 1 [•••] AR Engine Introduction. The tutorial aims to introduce the basic concepts of AR engines such as Unity and/or Unreal that are required to start developing AR applications. If the local study program does not cover this topic in any other mandatory modules before, we recommend to include this generic, non-AR-focused introduction.

Tutorial $2[\bullet \cdot$ Modelling AR UI/UX. Three-steps paper-based activity for brainstorming ideas of AR applications. It includes modeling the audience, the message and the worlds to answer the questions of who are the users, what is the main concept or purpose, and what should happen in the application.

Tutorial $3[\cdots \cdots]$ New AR Product Development. In this tutorial, learners should start thinking from a customer perspective. They should understand customer needs and organize them with social requirements engineering processes and tools on the Web. Basics of markets, minimal viable products and sales are introduced. Advanced tools like the Requirements Bazaars ${ }^{49}$ and the House of Quality can be utilized in practical sessions.

Tutorial 4 [••] Rapid Prototyping and Authoring. The tutorial provides practical experience in rapid prototyping using different AR authoring tools, such as Wikitude, InVision, Dart, Proto.io.

Tutorial 5 [•..•] Markers. The tutorial provides practical instruction on creating marker-based AR experience, including creation and packaging for AR engines and deploying to devices.

Tutorial $6[\bullet] 3 D$ modelling. The tutorial combines presentation, demonstrations and assisted experimentation to teach the fundamentals of 3D modelling and covers modelling, rigging, texturing/materials/lighting, animating, and exporting to AR engines for real-time application.

Tutorial $7[\bullet] 3 D$ scans, wrapping, \& animation. The tutorial guides through the construction of a $3 \mathrm{D}$ character by $3 \mathrm{D}$ scanning of a human, converting it into a low-poly 3D model, cleaning the geometry and textures, and animating it to be ready for an AR application.

Tutorial 8 [•.••] Spatial Mapping. The tutorial covers general features of Hololens spatial mapping technology, its functions and limitations, Hololens setup guide, indoor spatial mapping tryout, and processing the resultant map in a 3D modeling software.

Tutorial 9 [・..•] Gaze Interaction. This tutorial includes a detailed presentation of the concept of Gaze interaction and how gaze interaction is used to select objects. The tutorial will teach how to implement gaze interaction using prewritten scripts provided by the MixedRealityToolkit.

Tutorial 10 [・..•] Gesture Interaction. The tutorial explains how to add gesture recogniser, add tap events, manage with hands visible and tracked by Hololens, and gives a framework for deeper understanding of hand tracking and gaze outputs.

Tutorial 11 [•..•] Voice Interaction. The tutorial describes the basics of how spatial sound can add realism, direct the users gaze, and provide gesture feedback by creating simple voice commands and sounds of AR objects interaction in AR engines, such as Unity.

4.2.2 Skills Mapping for Foundations. When looking at the skills mapping for the undergraduate course (Table 2), the foundations in AR, the following picture emerges. The course should set strong emphasis on rapid prototyping, interaction technologies, and AR development skills plus platform specific skills and specific programming languages. Most of the practical tutorials cover multiple skill groups and require multiple lectures to be completed prior to the tutorial. At the same time, its lectures cover all skills areas (mostly through single skills, though). It should not, however, cover advanced advanced computing skills and machine learning (advanced data analysis and AI skills).

\footnotetext{
${ }^{49}$ https://requirements-bazaar.org
} 


\begin{tabular}{|c|c|c|c|c|c|c|c|c|c|c|c|c|}
\hline Unit & 1 & 2 & 3 & 4 & 5 & 6 & 7 & 8 & 9 & 10 & 11 & 12 \\
\hline$[\cdots]$ L1: Introduction to AR & + & & & & & & & & & & & \\
\hline [....] L2: Human Perception \& Processing & & & & & & & & & & ++ & & \\
\hline [•...] L3: Technology Overview & ++ & ++ & & & & & & & & & ++ & \\
\hline [ •. ] L4: Experience Engineering & & & & & & + & & & & ++ & & \\
\hline […] L5: HCI evaluation methodologies & & & & & & & & & & & & ++ \\
\hline$[\cdots \cdot . \cdot]$ L6: Spatial Computing & & & & & + & & & & ++ & ++ & & \\
\hline [ • ] L7: Software Development Methods & & + & & & & & & & & ++ & & \\
\hline [ • ] L8: Geometric Algebra & & & & & & ++ & & & & & & \\
\hline [ • ] L9: Storytelling & & & & & & & & & & ++ & & \\
\hline [ • ] L10: Careers in AR & & & & & & & & & & & & \\
\hline [ • ] L11: Design Inspiration & & & & & & + & & & & ++ & & \\
\hline [ •. ] L12: Research Directions & & & & & & & & & & & & \\
\hline$[\cdots . \cdot]$ T1: AR Engine Introduction & ++ & & & & & & & & & & & \\
\hline [・• ] T2: Modelling AR UI/UX & & & & & & & & & & & & ++ \\
\hline$[\cdots .$.$] T3: New AR Product Development$ & & & & & & & & & & & & ++ \\
\hline [ •. ] T4: Rapid Prototyping and Authoring & & ++ & & & & & & & + & ++ & & \\
\hline$[\cdots \cdot$ T5: Markers & ++ & ++ & & & ++ & & & & + & & & \\
\hline [ •. ] T6: 3D modelling & & & & & & ++ & & & & ++ & & \\
\hline [ - ] T7: 3D scan, wrap, \& animate & ++ & & & & ++ & ++ & & & & & & \\
\hline [•...] T8: Spatial Mapping & ++ & ++ & ++ & & + & & & & + & & + & \\
\hline$[\cdots . \bullet]$ T9: Gaze Interaction & ++ & ++ & ++ & & & & & & ++ & & + & \\
\hline$[\cdots .$.$] T10: Gesture Interaction$ & ++ & ++ & ++ & & & & & & ++ & & + & \\
\hline$[\cdots \cdots]$ T11: Voice Interaction & ++ & ++ & ++ & & & & & + & ++ & & + & \\
\hline
\end{tabular}

Table 2: Skills mapping (foundations course). Columns 1-12 refer to the groups of 'hard' skills, section 4.1.

4.2.3 Lesson Plan for Advanced. The advanced course follows the software development cycle from inception, to implementation, to validation. It adds design thinking and UX guidelines, as well as advanced storytelling. The implementation-focused technologies advance from the foundational course to cover spatial understanding (on top of spatial mapping), abstraction for cross-platform/multiuser/multi-device support, artificial intelligence dialog understanding, openCV foundations, wearable technology and making things talk, and volumetric video capture. Finally, evaluating AR introduces the methodologies available for verifying and validating applications. Insights into specialist application areas and job perspectives will help students to sharpen their skills set.

Lecture 1 [・.•] Introduction to Advanced AR. The lecture introduces the key concepts of AR, such as a modern definition of AR, concepts of augmentation, detectables, tracking systems, and sensors. The lecture also covers key standard AR architectures and presents examples of AR applications in different areas.

Lecture $2[\cdots \cdots]$ AR UX. Lecture about how AR apps are created from the design point of view, how to make them effective and scalable, both mobile and Head Mounted Display (HMD)-based. It covers user experience engineering processes, spatial user interface guidelines and design principles, and how modern usability methods can be used to evaluate AR products.

Lecture $3[\bullet \cdot$ AR Storytelling. This lecture describes the meaning of storytelling, its importance for AR apps, and the components of storytelling. The lecture also covers the computational approaches for storytelling: finite state machines and story maps. Several examples of AR apps and games that utilize storytelling to outline specific methods and techniques are discussed.

Lecture $4[\bullet \cdot$ AR Research Directions. The lecture covers the most recent trends, vision, and the challenges in AR and in related the areas, and specific application areas such as technology-enhanced learning, Industry 4.0, building information modeling, or AEC architecture, engineering, and construction.

Lecture $5[\bullet$ ] Wearable Computing. An introduction to the field of Wearable Computing, the practice of inventing, designing, building, or using body-worn computational and sensory devices, the functions and applications of wearable computers, design principles, and the underlying materials and technologies.

Lecture 6 [•.•] Computer Vision. This lecture covers key algorithms for motion tracking, object detection, and pose estimation. It looks at SDKs capabilities and existing open source solutions for features detection, description, and matching, also covering camera model and lens calibration.

Lecture $7[\bullet]$ AI dialog understanding. Some say that AI assistants are the key ingredient in future (spatial) user interfaces, so this lecture reviews what the area of artificial intelligence has to give to the cause, also reviewing the research history of relevant technologies and services for speech recognition and generation, machine translation, and dialogue understanding.

Lecture $8[\bullet \cdot$ AR Applications and Industry Cases. This lecture is suited for inviting local industry to showcase apps and projects, so as to demonstrate to students what is possible in a professional context, as well as offering opportunities for networking. 
Lecture $9[\cdots \cdot \bullet]$ Evaluation. The lecture provides an overview on suitable methodologies for evaluating AR applications, distinguishing between primary and secondary user experience metrics that can be used to evaluate and improve AR products. It introduces specialist evaluation methods such as SPINE, a new universal method to evaluate the usability of spatial user interfaces. Moreover, background on statistics theory is added where needed.

Lecture $10[\bullet \cdot$ Spatial Audio. This lecture covers an introduction to audio displays, how sound is captured or generated, including the relevant production pipelines and toolkits, working principles for human audio processing like interaural time differences and spectral filtering, production principles like head-related transfer functions.

Lecture $11[\bullet \cdot]$ Haptics. This lecture covers the design of haptic interaction with holograms and integrating force feedback generated within multimodal AR experience, using a variety of devices (e.g., wearable and hand-held).

Lecture $12[\bullet \cdot$ Mobile AR. This lecture covers mobile platforms, discussing the available frameworks and UX design principles for this form factor.

Lecture $13[\bullet \cdot$ Web $A R$. This lecture covers approaches for the web (WebXR, a-frames, three.js, etc.).

Lecture $14[\bullet]$ Volumetric Video. This lecture covers volumetric video as a novel format of interactive media that allows to bring live action content into AR. It introduces the content creation pipeline including multiview capture and other sensors, basic computer vision tasks such as segmentation/keying, calibration and colour correction, as well as typical 3D reconstruction approaches such as shape-from-silhouette, visual hulls and structure-from-motion. Data representation, coding and streaming as dynamic 3D meshes or point clouds are covered next. Finally, immersive visualization, interaction and application scenarios are outlined.

Lecture $15[\bullet]$ Advanced Computer Graphics for AR. This lecture covers such advanced graphics techniques as low level OpenGL Shader and GPU programming, photorealistic and non-photorealistic rendering, light capture and relighting techniques, visualization methods, occlusion, graphics optimization and parallelization, $\mathrm{x}$-ray techniques, and the latest graphics research methods in AR.

Tutorial $1[\bullet \cdot$ AR UX. This tutorial includes reviewing example AR applications and discussing features and issues.

Tutorial $2[\bullet \cdot]$ Designing AR Workflows. This tutorial provides practical guidelines on the workflows used in prototyping and creating AR apps. It helps to create a more coherent encompassing design and production workflow for AR.

Tutorial 3 [••••] Collaboration and Sharing. Synchronous collaboration in Mixed Reality is possible in a shared virtual environment. This means that the shown 3D objects are synchronized between the participants and changes are broadcast in real-time. This tutorial is introducing a communication library for online games (like Photon) as a tool for creating sharing and collaboration experiences in mixed reality with a running example of a two person checkers game in mixed reality.

Tutorial $4[\bullet]$ Making Things Talk. This tutorial guides through the steps to link an AR project to microcontroller devices through WiFI and Bluetooth. It demonstrates the full development pipeline from, for example, an Arduino sketch to a Unity client that interacts with HoloLens
Tutorial 5 [•.••] Computer Vision. The tutorial practices the key computer vision algorithms in OpenCV.

Tutorial $6[\bullet]$ AI dialog understanding. The tutorial guides through the steps required to create a responsive 3D character in Unity using IBM's Watson APIs, starting with explaining how to set up the needed cloud accounts, instantiating the according services, and how to configure service credentials in Unity. It shows how to work with dialogue understanding, speech to text, and text to speech with an example holographic AI.

Tutorial $7[\bullet$ ] Careers in AR. Following an introduction, in a series of interactive exercises, participants explore what careers are possible and which ones they might be interested in.

Tutorial $8[\bullet]$ Volumetric video capture. The tutorial guides the students through setting up and using the camera rigs and software required for capturing volumetric video. It guides them through the post processing tool chain and shows how to integrate results into the AR app.

Tutorial 9 [•••] Spatial Understanding. An overview of spatial understanding, demonstration of the solvers' use and how these control the way objects behave in the playspace.

Tutorial $10[\bullet \cdot$ WebXR. WebXR is a collection of standards that support rendering of $3 \mathrm{D}$ scenes in $\mathrm{AR}$ on eyeglasses as well as handheld devices. This tutorial guides students through their first AR app built with web technology.

Tutorial $11[\bullet \cdot$ AR Evaluation. This tutorial uses Google forms for administering three evaluation instruments for AR, namely SPINE, TAMARA, and TRUST, to conduct a test evaluation jointly with the participants. An associated Overleaf LATEXdocument uses knitr to integrate the $\mathrm{R}$ code required for the analysis, presenting results live and on click of a button.

Tutorial $12[\bullet]$ AR portals using shaders. The tutorial is a simple introduction to Unity shaders, demonstrating how two shaders can be used to mask $3 \mathrm{D}$ objects in a way that they become visible only when looking through a portal window.

Tutorial $13[\bullet]$ Geo-Location based AR. The tutorial shows how to work with GPS in AR, to create Pokemon-go like apps.

Tutorial $14[\bullet \bullet]$ Performance profiling. Monitoring and optimising the performance of an AR application is important to ensure a pleasant user experience. Performance profiling of AR applications is not only concerned with the responsiveness of the app. Instead, developers need to look at further measures like the application's framerate. This tutorial introduces performance metrics, performance profiling tools and performance optimization.

Tutorial $15[\bullet \bullet]$ AR Unit Testing. Unity, for example, ships with a test runner that uses the NUnit framework. NUnit is part of the xUnit testing framework collection. It is the official solution by Unity and is actively used by an increasing number of people in the development of modules for the package manager that was introduced in Unity 2018. This tutorial explores the possibilities of the NUnit framework.

4.2.4 Skills Mapping for Advanced. Regarding the skills mapping for the advanced AR course (Table 3), the following can be said. All skills groups cover more individual skills. Moreover, also the ones missing from the foundations course should be covered here (including advanced computer graphics and advanced data analysis and AI skills). 


\begin{tabular}{|c|c|c|c|c|c|c|c|c|c|c|c|c|}
\hline Unit & 1 & 2 & 3 & 4 & 5 & 6 & 7 & 8 & 9 & 10 & 11 & 12 \\
\hline$[\cdots \cdot]$ L1: Introduction to Advanced AR & ++ & & & & & & & & & & & \\
\hline$[\cdots \cdot \cdot]$ L2: AR UX & & & & & & & & & & ++ & & + \\
\hline [ • ] L3: AR Storytelling & & & & & & & & & & ++ & & + \\
\hline \multicolumn{13}{|l|}{ [ •• ] L4: AR Research Directions } \\
\hline [ • ] L5: Wearable Computing & ++ & ++ & & & & & & & ++ & & ++ & \\
\hline [....] L6: Computer Vision & & & & & ++ & & ++ & & ++ & & & \\
\hline [ - ] L7: AI dialog understanding & ++ & & ++ & & & & ++ & & ++ & & & \\
\hline \multicolumn{13}{|l|}{ [ •. ] L8: Applications \& Industry Cases } \\
\hline$[\cdots]$ L9: Evaluation & & & & & & & ++ & & & & & ++ \\
\hline [•• ] L10: Spatial Audio & ++ & & ++ & & & & & ++ & & & & \\
\hline [・・ ] L11: Haptics & & & & & & & & + & & + & & \\
\hline [••] L12: Mobile AR & ++ & ++ & ++ & & + & & & & & & + & \\
\hline$[\cdot \bullet]$ L13: Web AR & ++ & ++ & ++ & & & & & & & & & \\
\hline [• ] L14: Volumetric video & & & & & ++ & ++ & ++ & & & & & \\
\hline [ - ] L15: Advanced CG for AR & & ++ & & & & ++ & ++ & & & + & & \\
\hline [・ ] T1: AR UX & & & & & & & & & & ++ & & \\
\hline [ • ] T2: Designing AR Workflows & & & & & & & & & & ++ & & \\
\hline […] T3: Collaboration and sharing & ++ & & ++ & ++ & + & & & & & & & \\
\hline$[\cdot]$ T4: Making Things Talk & ++ & ++ & ++ & ++ & & & & & ++ & & ++ & \\
\hline [•...] T5: Computer Vision & & & & & ++ & & ++ & & ++ & & & \\
\hline [ • ] T6: AI dialog understanding & ++ & & & & & & ++ & & ++ & & & \\
\hline \multicolumn{13}{|l|}{ [ • ] T7: Careers in AR } \\
\hline [ • ] T8: Volumetric video capture & & & & & ++ & ++ & ++ & & & & & \\
\hline [....] T9: Spatial Understanding & ++ & & ++ & & ++ & & & & ++ & & & \\
\hline$[\cdot \bullet]$ T10: WebXR & ++ & ++ & ++ & & & & & & & & & \\
\hline [•. ] T11: AR Evaluation & & & & & & & ++ & & & & & ++ \\
\hline [ •. ] T12: AR portals with shaders & ++ & & ++ & & & ++ & & & & & & \\
\hline [ • ] T13: Geo-Location based AR & ++ & ++ & ++ & & & & & & & & ++ & \\
\hline$[\cdot \bullet$ ] T14: Performance profiling & ++ & ++ & & ++ & & & + & & & & ++ & \\
\hline [•• ] T15: Unit Testing and Integration & ++ & & & ++ & & & & & & & ++ & \\
\hline
\end{tabular}

Table 3: Skills mapping (advanced course).

Columns 1-12 refer to the groups of 'hard' skills, section 4.1.

\subsection{Learning Objectives}

With regards to the intended learning outcomes, we propose to break them down into three groups, namely knowledge, skills, and key competences. Knowledge and skills are directly related to the skills framework and lesson plans introduced above, whereas the key competences comprise the cross-cutting elements that are motivated rather by the chosen organisational format and assessment regime.

4.3.1 Intended Learning Outcomes for Foundations. The knowledge, skills and key competences for the Foundations course are presented below.

Knowledge: State the conceptual origins, advantages, and disadvantages of various methods used for solving problems for the given application domain of AR. Core topics include:

- 3D content acquisition and handling including 3D modelling, photogrammetry, animation, mesh optimisation

- Object recognition using image targets and and fiducial markers

- Environment mapping

- AR-specific interaction such as methods gaze, voice, gestures
Skills: Students should be able to:

- Brainstorm, review, and select use cases and match them to the range of AR toolkits and platforms available

- Develop iteratively, and in a team, an application utilising AR toolkits and platforms

- Apply AR-specific User-Centred Design and Software Engineering approaches

Key Competences: Based on the knowledge and skills acquired, students should be able to:

- Present technical work, a use case and project progress, either verbally or in written reports

- Enact a variety of roles in a technical project team, as determined by the requirements of agile project management approaches

- Plan projects and meet milestones

4.3.2 Intended Learning Outcomes for Advanced. The knowledge, skills and key competences for the Advanced course are presented below.

Knowledge: State the alternatives, pros, and cons of architecture, frameworks, components, and associated hardware required for 
solving problems in the given application domain of AR. Core topics include:

- AR User Experience Engineering, including methodological knowledge about design thinking for AR with a special focus on storytelling and storyboarding

- Develop versatility in AR-specific evaluation Methodologies for usability, user experience, technology acceptance

- Extended computer vision specialist knowledge in Spatial Understanding, low-level Computer Vision engineering, Volumetric video

- Deep specialisation in interaction technology including in Artificial Intelligence, cross-device/cross-platform Remoting and Collaboration, Wearable Computing

Skills: Students should be able to:

- Brainstorm, review, and select use cases and devise plans for realising them involving making appropriate choices regarding hardware, platform, software development environment, and development framework.

- Develop iteratively, and in a team, an application utilising the developed knowledge and skills in software engineering [Remove if lecture and practicals only, no independent study]

- Apply AR-specific user-centred design approaches and evaluation methodologies.

Key Competences: Based on the knowledge and skills acquired, students should be able to:

- Present technical work, a use case and project progress, either verbally or in written reports

- Enact a variety of roles in a technical project team, as determined by the requirements of agile project management approaches

- Plan projects and meet milestones

- Utilise verification, optimisation, and instrumentation methods

\subsection{Suggested Course Format and Options}

It is certainly up to each organisation implementing this curriculum to decide on whether to choose a classical face-to-face format, a blended form mixing online and face-to-face elements, or even a flipped classroom format, where lectures are streamed home and the contact time is used for collaboration and discussion.

As already explained with the lesson plans, the lectures are means of teaching the theoretical underpinnings of designing and developing AR applications, followed by practical sessions, in which students are assigned exercises involving practicing and further exploring material from the preceding lectures.

Lectures and tutorials sum up to a maximum of $22 \times 1.5$ hours for the foundations course and to a maximum of $29 \times 1.5$ hours for the advanced course, though different selections of optional lectures and tutorials may result in a lower number of contact hours here. In Europe, depending on the country, 1 credit point (ECTS point) equals between 25 and 30 hours of work for the students. 1 ECTS point equals 0.5 US points and 2 UK points. Ideally, the foundation course module aims for 7.5 ECTS points, same as the advanced course. This means they require a student engagement of between 187.5 and 225 hours. The timings fall into the following categories, but other splits are possible and elements of the courses can be stacked or distributed across semesters as needed: lectures, tutorials, guided independent study, preparing for assessments.

It is possible to space out the hours across courses. For example, one can imagine a scenario where there are lecture and practicals only, with assessment being merely based on attendance and success in the practicals, with a final exam concluding the course, asking, e.g., some discussion and understanding questions (for example, fantasy customer case: explain which interaction technique you propose and discuss why). This would give only the hours of 2 ECTS (1 US / 4 UK) points, if the preparation for an exam is included.

This makes particular sense, where courses are split into two parts, spaced out across semesters, with the following semester complementing the lectures and tutorials with a course project, where the hours of independent guided study are then put in in the inception, implementation, testing, and documentation of a team project. These project courses exist in many places, often open to all students from all routes. For example, in many places, the final thesis is guided by a thesis course, where students develop their own project and then write their final thesis about it. In other places, block courses are used to bundle lectures and tutorials into an intensive week, followed subsequently by the team project leading through the rest of the semester or by an intensive hackathon.

Alternatively, and we anticipate this will rather be the norm, the course can be delivered with lectures and tutorials spaced out over 12 weeks of study, with students working on small groups projects in parallel in a lab course, enhancing functionality as they learn about it in the tutorials as they go along. This then includes the guided independent study (and the preparation for the reviews), which makes up for the hours needed to justify 15 ECTS (7.5 credits in the US / 30 credits in the UK).

Assessment follows this rationale and is stackable. If aiming for 2 (1 US / 4 UK) ECTS points only, focusing on lectures and tutorials, we recommend to complement monitoring of attendance and success in the practicals, culminating with an exam at the end.

If aiming for a full 7.5 ECTS (3,75 US / $15 \mathrm{UK}$ ) points and including a project, the assessment should be designed to let the students experience the full life-cycle of a practical project, as they will face in industry or research, from design via implementation, testing, evaluation, demonstration, to documentation.

For example, selected lectures and tutorials from the lesson plans above were adopted in a schedule (see Figure 2) for a one week intensive course at the University of Klagenfurt, Austria, in summer 2019. The students were from study programs in technical as well as design-oriented subjects. Besides the lectures and tutorials, they also conducted a week-long group work on self-selected projects.

\subsection{Suggested Assessment Regimes}

If embedded in a 7.5 ECTS course, the assessment should be designed to best let the students experience the full lifecycle of a practical project, which they will face once they leave the university for industry or research. The experience will be gained from active design via implementation, testing, evaluation, demonstration, to documentation. As experience promotes self-directed learning and is based on the value of self-proposed projects, the assessment is 


\begin{tabular}{|c|c|c|c|c|c|}
\hline $\begin{array}{l}\text { Wednesday } \\
12.06\end{array}$ & $\begin{array}{l}\text { Thursday } \\
13.06\end{array}$ & $\begin{array}{l}\text { Friday } \\
14.06\end{array}$ & \begin{tabular}{|l|} 
Monday \\
17.06
\end{tabular} & \begin{tabular}{|l} 
Tuesday \\
18.06
\end{tabular} & \begin{tabular}{|l} 
Wednesday \\
19.06
\end{tabular} \\
\hline $\begin{array}{l}10.00-12.00 \\
\text { Introduction and } \\
\text { Overview } \\
\text { Perception }\end{array}$ & $\begin{array}{l}13.00-15.00 \\
\text { Software } \\
\text { Development } \\
\text { with Unity and } \\
\text { MRTK }\end{array}$ & $\begin{array}{l}13.00-14.30 \\
\text { AR Concepts and } \\
\text { Technologies } \\
\text { Milestone } 1\end{array}$ & $\begin{array}{l}12.00-14.00 \\
\text { Gaze, Voice, } \\
\text { Gesture } \\
\text { Milestone 2 }\end{array}$ & $\begin{array}{l}8.00-10.00 \\
\text { Storytelling and } \\
\text { Gamification }\end{array}$ & $\begin{array}{l}8.00-10.00 \\
\text { Final Demos und } \\
\text { Presentations } \\
\text { Q \& A }\end{array}$ \\
\hline $\begin{array}{l}14.00-16.00 \\
\text { Unity and } \\
\text { Hololens } \\
\text { Introduction } \\
\mathrm{HCl} \\
\text { Methodologies }\end{array}$ & $\begin{array}{l}15.00-18.00 \\
\text { Practical } \\
\text { Exercises }\end{array}$ & & $\begin{array}{l}16.00-18.00 \\
\text { Colloquium: } \\
\text { Teaching with AR } \\
\text { and teaching AR }\end{array}$ & $\begin{array}{l}12.00-14.00 \\
\text { Design } \\
\text { Inspirations and } \\
\text { Research } \\
\text { Directions }\end{array}$ & \\
\hline
\end{tabular}

Figure 2: Example bootstrapped course.

best if based $100 \%$ on a self-defined project, which can be done individually or in small groups[3]. Such approach is a standard Project Based Learning methodology that increases the motivation and interest of the students [14] [4].

The overall assessment methodology begins with project ideas that are discussed and refined in an iterative process to make sure they fit in scope and magnitude. Approved projects are defined in a document of up to one page. The smaller assignments in the middle of the course (project idea, review of mockups, etc.) help the average student stay active. The marking scheme itself then varies according to local requirements. For example, we have identified the following four different regimes:

- Delivery: report, video and the code. Assessment criteria: creativity/originality $30 \%$, quality/results $30 \%$, and report $40 \%$.

- Assessment is based on the work of the team project. The assessment is split into three interim reviews (10\% each) and one final assessment of the course work (70\%).

- Four reviews are scheduled over the course of the project work. The first three reviews are targeting technical and development issues, while the last review is planned as a public review akin to a business pitch. Every review contributes equally to the final grade.

- Review 1 (emphasis: market analysis, technology survey)

- Review 2 (emphasis: UI mockups, tests)

- Review 3 (emphasis: demo, tests, documentation)

- Final course project (code repository, user documentation, walkthrough video, report)

- Three assignments are planned to move from scene to interactive scene to application.

- Assignment 1: Building a Virtual Reality scene (after 5 weeks, $30 \%$ )

- Assignment 2: Developing an Interactive Scene (after 4 more weeks, $30 \%)$

- Assignment 3 (project and presentation): Developing a handheld mobile AR application +10 minute presentation of a research paper in class (end of the course, 40\%)

Feedback should be given in class as students attempt practical problems. The project builds on the knowledge from the lectures and workshops, and the feedback given during classes will inform the student in their attempts on the final project. To provide formative feedback, students will be asked to present their project ideas (proposal elevator pitch), give an interim progress report (presentation), and demo.

\subsection{Open Education Resources and Reading List}

The AR-FOR-EU consortium initiated the writing of an open textbook, as comprehensive Open Educational Resource (OER) to cover the foundation for AR curricula in Higher Education. The book is Open Source with an open community of contributors, sources on GitHub ${ }^{50}$, thus allowing for quick update cycles to avoid the typical high tech trap, risking to be outdated already when going off to print. The book's compiled version is available here: https://codereality.net/ar-for-eu-book/. The community particularly welcomes students and brings them together with established researchers in the field for collaboration and quality assurance. Many chapters in the book map directly to lectures and tutorials presented.

CC-BY Slide decks are available for the foundation ${ }^{51}$ and for the advanced ${ }^{52}$. Open access lecture recordings and two sample moodle courses with most of the units listed above are available at Code Reality: Learn ${ }^{53}$. Additional lectures can be found here ${ }^{54}$ (Mark Billinghurst) and here ${ }^{55}$ (Dieter Schmalstieg).

With regards to a short reading list, we recommend the following works:

- The Open Augmented Reality Teaching Book, AR-FOR-EU consortium, CodeReality.net ${ }^{56}$ [5]

- Speicher, M., Hall, B., Nebeling. M. (2019): What is Mixed Reality?, In: CHI 2019, May 4-9, 2019, Glasgow, Scotland, UK [30]

- Billinghurst, M., Clark, A., and Lee, G. (2015). A survey of augmented reality. In: Foundations and Trends in $\mathrm{Hu}-$ man-Computer Interaction, 8(2-3), 73-272. [9]

- Schmalstieg, D., Höllerer, T. (2016). Augmented reality: principles and practice, Boston: Addison-Wesley. [28]

- Furht, B. (2011): Handbook of Augmented Reality, Springer. [18]

- Craig, A. (2013). Understanding Augmented Reality: Concepts and Applications, Morgan Kaufmann. [11]

- Fominykh, M., Bilyatdinova, A., Koren, I., Jesionkowska, J., Karsakov, A., Khoroshavin, A., Klamma, R., Klimova, A., Molka-Danielsen, J.; Rasool, J., Smith, C. H., and Wild, F. (2019): Existing Teaching Practices and Future Labour Market Needs in the Field of Augmented Reality, AR-FOR-EU consortium, CodeReality.net ${ }^{57}$ [16]

\section{DISCUSSION AND RECOMMENDATIONS}

Our review of the state of the art finds that AR curricula are offered primarily in Higher Education and CS departments. However, there is an ever-increasing interest for an AR curriculum in other disciplines such as Engineering, Medicine, Science, Arts and Design.

\footnotetext{
${ }^{50}$ https://github.com/klamma/ar-for-eu-book

${ }^{51} \mathrm{https} / / /$ drive.google.com/drive/folders/1Arh0M8A0J3OZSxd0DWzlYhN8F3tOYajn? usp=sharing

${ }^{52}$ https://drive.google.com/drive/folders/16nQ60GArRUhejgvZ8QWDlbTzefExPzOs? usp=sharing

${ }^{53}$ https://codereality.net/learn/

${ }^{54}$ https://www.slideshare.net/marknb00/

${ }^{55}$ https://arbook.icg.tugraz.at/

${ }^{56} \mathrm{https} / /$ codereality.net/ar-for-eu-book/

${ }^{57}$ https://codereality.net/report/
} 
This is certainly due to the interdisciplinary potential we identify above. To this end, it would certainly help to further investigate the model curriculum presented in this paper in terms of target groups, and to assess its applicability outside of CS. In fact, several authors of this paper have drawn from their extensive experience and taught such proposed course modules across various disciplines. Making courses available to design, arts, education, life sciences, and humanities has been simultaneously challenging and rewarding, while especially effective when lectures and tutorials have been delivered 'blocked' or compressed into short week-long courses.

Further investigation into different instruction methods, modes of delivery, and assessments will prove valuable, when brought into alignment with best practices and fleshed out via case studies. This would be particularly useful for ACM and IEEE CS program curriculum working groups.

Future AR education should take AR experiences and actual AR teaching environments into account. In so doing, learners can directly experience interaction, display, and tracking technologies while using augmented teaching spaces. The design of the user experience, as well as a comprehensive understanding of spatial computing will benefit, especially if feedback is given directly from users within the intended delivery environment. An example of such a future teaching environment is VIAProMa ${ }^{58}$, a collaborative mixed reality environment for immersive analytics [23]. Learners benefit from training, agile project management practices as well as collaboration within such mixed reality environments. Resnyansky et al. give further insights into how AR can be utilised for CS education [26]. In particular, they note that AR can be combined with Tangible User Interface techniques in order to visually teach programming concepts. They catalogue an overview of current tools for teaching programming concepts, and also discuss ways in in which AR can be used to further improve learning.

$\mathrm{AR}$ is a topic that relies on using special purpose hardware: smart glasses, depth cameras, and sensors. These are normally available in research labs, but not as often in teaching labs. In a time when remote teaching has become essential, new solutions for hardware accessibility are needed. Renting expensive special purpose hardware as well as ordering online or shipping smaller and cheaper components are viable options. Additionally, using common technology such as mobile phones with cameras, web cameras, or low-end HMDs are potential alternatives. Also of note are platforms such as Amazon Web Services, which offers remote app streaming services ${ }^{59}$, alongside remote desktop services such as Citrix $^{60}$, which ensures remote access to relevant hardware and software for teaching the curriculum, which potentially opens course participation to a global audience.

Given the fact that AR technology is advancing faster than legislation addressing any relevant ethical implications, the AR model curriculum might also cover ethical issues. This can increase awareness as well as empower students in so far as what they wish to see through the technology, what data becomes accessible to whom, and how the technology can affect society as a whole. While currently, in many places, such issues are covered by ethics clearance processes which are attached to project work (and critical analysis

\footnotetext{
${ }^{58}$ https://github.com/rwth-acis/VIAProMa

${ }^{59}$ For example, https://aws.amazon.com/appstream2/

${ }^{60}$ https://www.citrix.com/en-gb/products/citrix-virtual-apps-and-desktops/
}

requirements for course work), any future curriculum might speak to concerns for AR privacy, security and ethical design.

Given a plethora of online AR curricula designs, one must take into account the internationalisation of the curriculum, while recognizing the global spectrum of participants. Consequently, educators should incorporate international, intercultural, and global aspects, at least as far as the AR context of the curriculum, learning outcomes, assessment tasks, teaching methods, and support services of the study program [21] are concerned. Given the practical perspective of AR courses [22], internationalisation of the curriculum is an important part of the periodic and critical review of the curriculum. Since the AR curriculum is at early stages and this paper is the first attempt to highlight and summarize learning outcomes, assessment strategies and content skills, any future work for the AR curriculum will benefit by assessing the extent to which an AR curriculum is internationalised as well as whether there are cross-cultural specificities to take into account. Common aspects might be identified across various curricula worldwide, however, with different modes of delivery and the engagement of global participation, any curriculum should be reviewed in terms of the following: rationale and state of internationalisation (and according arrangements specificities), evaluation and feedback mechanisms provided to take stock of internationalisation, benchmarking feedback by global participants on different elements of the course blueprints, and benchmarking feedback from other stakeholders (such as professional associations or industry).

\section{CONCLUSIONS}

With this contribution, the ACM ITiCSE 2020 Working Group 5 for Developing a Model Augmented Reality Curriculum [17] concludes its efforts to improve education in the new and rapidly developing AR discipline. The proposed skills framework and detailed blueprint, which covers two course modules, should provide institutes of higher education with an excellent overview of the present needs of the AR market as well as useful guidelines for the development of much needed new courses, including the relevant teaching materials.

A needs continuously evolve, however, we would therefore like to recommend to SIGCSE to install a standing expert commission within the ACM (possibly in collaboration with the IEEE Computer Society). Such commission would revisit this model curriculum on a regular, annual basis, to assess whether its relevance to the state of art, as well as to propose updates and upgrades.

With our proposal, it is now possible to integrate AR foundations and advanced courses into curricula recommendations in regards full programs, such as an updated version of the ACM and IEEE Computer Society curricula recommendations for computer science, computer engineering, and information technology. This could be, for example, an alternative on the level of Internet-of-things or Mobile Applications in Information Technology, Embedded Systems in Computer Engineering, or as electives in CS (in particular in $\mathrm{HCI}$ or Graphics and Visualisation). Further work on recommendations regarding particular delivery modes (online, face-to-face, or blended; independent study; group work; etc.) which are suitable or challenging for teaching different AR skills would be invaluable for instructors. 
We also propose a web-based course builder and assessment tool to be developed in the near future. With such a tool, directors of studies and lecturers can select options and alternatives for lectures as well as practicals, checked against the skills portfolio they are targeting. This allows customization of duration and coverage, and, in consequence, credit points to be awarded as well as associated assessment regimes.

\section{ACKNOWLEDGMENTS}

The working group has been supported by projects AR-FOR-EU, ARETE, and FANTASIA that are funded by the European Commission.

\section{REFERENCES}

[1] ACM. 2016. ACM Computer Engineering recommendations 2016: Computer Engineering Curricula 2016: Curriculum Guidelines for Undergraduate Degree Programs in Computer Engineering.

[2] ACM. 2017. ACM Information Technology recommendations 2017: Information Technology Curricula 2017: Curriculum Guidelines for Baccalaureate Degree Programs in Information Technology.

[3] Heidi Andrade. 2019. A Critical Review of Research on Student Self-Assessment Frontiers in Education 4, 87 (2019). https://doi.org/10.3389/feduc.2019.00087

[4] Heidi Andrade and Anna Valtcheva. 2009. Promoting Learning and Achievement Through Self-Assessment. Theory Into Practice 48, 1 (2009), 12-19. https://doi. org/10.1080/00405840802577544

[5] AR-FOR-EU. 2020. The Open Augmented Reality Teaching Book. AR-FOR-EU consortium. https://codereality.net/ar-for-eu-book/

[6] José Maria Ariso. 2017. Augmented reality. De Gruyter, Berlin.

[7] Evan Barba, Yan Xu, Blair Maclntyre, and Tony Tseng. 2009. Lessons from a Class on Handheld Augmented Reality Game Design. In Proceedings of the 4th International Conference on Foundations of Digital Games (FDG '09). Association for Computing Machinery, New York, NY, USA, 2-9. https://doi.org/10.1145/ 1536513.1536525

[8] James Bennett and Amanda Murphy. 2020. Skills for immersive experience creation StoryFutures Academy, London.

[9] Mark Billinghurst, Adrian Clark, and Gun Lee. 2015. A Survey of Augmented Reality. Found. Trends Hum.-Comput. Interact. 8, 2-3 (March 2015), 73-272. https://doi.org/10.1561/1100000049

[10] Marcus Carter and Ben Egliston. 2020. Ethical Implications of Emerging Mixed Reality Technologies. https://ses.library.usyd.edu.au/handle/2123/22485

[11] Alan B. Craig. 2013. Understanding Augmented Reality: Concepts and Applications (1st ed.). Morgan Kaufmann Publishers Inc., San Francisco, CA, USA.

[12] ACM CS2013. 2013. Computer Science Curricula 2013. Curriculum Guidelines for Undergraduate Degree Programs in Computer Science.

[13] Chris Edwards. 2020. ETSI pushes for standards in augmented reality. https://eandt.theiet.org/content/articles/2020/05/etsi-pushes-for-standardsin-augmented-reality/

[14] Heidi J. C. Ellis and Richard Mitchell. 2004. Self-grading in a project-based software engineering course. In 17th Conference on Software Engineering Education and Training, 2004. Proceedings. 138-143.

[15] Brian Feldman. 2016. Yes, You Can Catch Pokémon at Auschwitz. https://nymag. com/intelligencer/2016/07/yes-you-can-catch-pokemon-at-auschwitz.html
[16] Mikhail Fominykh, Anna Bilyatdinova, Istvan Koren, Joanna Jesionkowska, Andrey Karsakov, Aleksandr Khoroshavin, Ralf Klamma, Alexandra Klimova, Judith Molka-Danielsen, Jazz Rasool, Carl H Smith, and Fridolin Wild. 2019. Existing Teaching Practices and Future Labour Market Needs in the Field of Augmented Reality. Retrieved June 19, 2020 from https://codereality.net/report/

[17] Mikhail Fominykh, Fridolin Wild, Ralf Klamma, Mark Billinghurst, Lisandra S. Costiner, Andrey Karsakov, Eleni Mangina, Judith Molka-Danielsen, Ian Pollock, Marius Preda, and Aljosa Smolic. 2020. Developing a Model Augmented Reality Curriculum. In Proceedings of the 2020 ACM Conference on Innovation and Technology in Computer Science Education (ITiCSE '20). Association for Computing Machinery, New York, NY, USA, 508-509. https://doi.org/10.1145/3341525.3394991

[18] Borko Furht. 2011. Handbook of Augmented Reality. Springer Publishing Company, Incorporated.

[19] Hired. 2020. State of Software Engineers. Hired.

[20] Alexandra Klimova, Anna Bilyatdinova, and Andrey Karsakov. 2018. Existing Teaching Practices in Augmented Reality. Procedia Computer Science 136 (2018), 5-15. https://doi.org/10.1016/j.procs.2018.08.232 7th International Young Scientists Conference on Computational Science, YSC2018, 02-06 July2018, Heraklion, Greece.

[21] Betty Leask. 2015. Internationalising the Curriculum. Routledge, Abingdon.

22] Betty Leask and Christopher Bridge. 2013. Comparing internationalisation of the curriculum in action across disciplines: theoretical and practical perspectives. Compare: A fournal of Comparative and International Education 43, 1 (2013), 79-101. https://doi.org/10.1080/03057925.2013.746566

[23] Kim Marriott, Falk Schreiber, Tim Dwyer, Karsten Klein, Nathalie Henry Riche, Takayuki Itoh, Wolfgang Stuerzlinger, and Bruce H. Thomas. 2018. Immersive Analytics. Springer, Cham.

[24] Will Markow Matthew Walsh and Scott Bittle. 2019. Visualizing the Future: Demand for 3D Graphics and Real-time 3D Across the Economy. Burning Glass Technologies / Epic Games.

[25] Vinh T. Nguyen, Kwanghee Jung, and Tommy Dang. 2019. Creating Virtual Reality and Augmented Reality Development in Classroom: Is it a Hype? In 2019 IEEE International Conference on Artificial Intelligence and Virtual Reality (AIVR). 212-217.

[26] Dmitry Resnyansky, Emin İbili, and Mark Billinghurst. 2018. The Potential of Augmented Reality for Computer Science Education. In 2018 IEEE International Conference on Teaching, Assessment, and Learning for Engineering (TALE). 350356

[27] Nancy Rodriguez. 2016. Teaching Virtual Reality with Affordable Technologies. In Human-Computer Interaction. Theory, Design, Development and Practice, Masaaki Kurosu (Ed.). Springer International Publishing, Cham, 89-97.

[28] Dieter Schmalstieg and Tobias Höllerer. 2016. Augmented Reality: Principles and Practice. Addison-Wesley. https://books.google.co.uk/books?id=Y4r-ngEACAAJ

[29] M. Singh and M. P. Singh. 2013. Augmented Reality Interfaces. IEEE Internet Computing 17, 6 (2013), 66-70.

[30] Maximilian Speicher, Brian D. Hall, and Michael Nebeling. 2019. What is Mixed Reality? In Proceedings of the 2019 CHI Conference on Human Factors in Computing Systems (CHI '19). Association for Computing Machinery, New York, NY, USA, 1-15. https://doi.org/10.1145/3290605.3300767

[31] Mark Sullivan. 2020. 'Face search' creeps people out. But it still has a future-in $A R$. https://www.fastcompany.com/90463340/face-search-freaks-people-outbut-it-still-has-a-future-in-ar

[32] SuperData. 2020. SuperData XR Q1 2020 Update. Retrieved April 27, 2020 from https://www.superdataresearch.com/blog/superdata-xr-update

[33] Fridolin Wild. 2020. 1589-2020 - IEEE Standard for Augmented Reality Learning Experience Model. Retrieved April 27, 2020 from https://standards.ieee.org/ standard/1589-2020.html 